\title{
Variationally Constrained Numerical Solution of Electrical Impedance Tomography
}

\author{
Liliana Borcea*, Genetha Anne Gray ${ }^{\dagger}$ and Yin Zhang ${ }^{\ddagger}$
}

December 17, 2002

\begin{abstract}
We propose a novel, variational inversion methodology for the electrical impedance tomography problem, where we seek electrical conductivity $\sigma$ inside a bounded, simply connected domain $\Omega$, given simultaneous measurements of electric currents $I$ and potentials $V$ at the boundary. Explicitly, we make use of natural, variational constraints on the space of admissible functions $\sigma$, to obtain efficient reconstruction methods which make best use of the data. We give a detailed analysis of the variational constraints, we propose a variety of reconstruction algorithms and we discuss their advantages and disadvantages. We also assess the performance of our algorithms through numerical simulations and comparisons with other, well established, numerical reconstruction methods.
\end{abstract}

\section{Introduction}

Electrical properties such as the electrical conductivity $\sigma$ and the electric permittivity $\epsilon$, determine the behavior of materials under the influence of external electric fields [58]. Let us consider a bounded, simply connected set $\Omega \subset \mathbb{R}^{d}$, for $d \geq 2$ and, at frequency $\omega$, let $\gamma$ be the complex admittivity function

$$
\gamma(\mathbf{x}, \omega)=\sigma(\mathbf{x})+i \omega \epsilon(\mathbf{x}), \text { where } i=\sqrt{-1} .
$$

The electrical impedance is the reciprocal of $\gamma$ and it measures the ratio between the electric field and the electric current at location $\mathrm{x} \in \Omega$. Electrical Impedance Tomography (EIT) is the inverse problem of determining the impedance in the interior of $\Omega$, given simultaneous measurements of direct or alternating electric currents and voltages at the boundary $\partial \Omega$ (i.e., the Neumann to Dirichlet or Dirichlet to Neumann map).

In this paper, we consider direct electric current excitations (i.e., $\omega=0$ ) and we seek to image the electrical conductivity function $\sigma(\mathbf{x})$ inside $\Omega$. Different materials display different electrical properties (see for example $[10,65,84]$ ) so a map of $\sigma(\mathbf{x})$, for $\mathbf{x} \in \Omega$, can be used to infer the internal structure in $\Omega$. Due to this fact, EIT is an imaging tool with important applications in fields such as medicine, geophysics, environmental sciences and nondestructive testing of materials. Examples of medical applications of EIT are the detection of pulmonary emboli [27,52,54], monitoring of apnea [2], monitoring of heart function and blood flow [44,56] and breast cancer detection [27].

\footnotetext{
${ }^{*}$ Computational and Applied Mathematics, MS 134, Rice University, 6100 Main Street, Houston, TX 770051892.(borcea@caam.rice.edu)

${ }^{\dagger}$ Sandia National Labs, P.O. Box 969, MS 9217, Livermore, CA 94551-0969. (gagray@sandia.gov)

${ }^{\ddagger}$ Computational and Applied Mathematics, MS 134, Rice University, 6100 Main Street, Houston, TX 770051892.(zhang@caam.rice.edu)
} 
In geophysics and environmental sciences, EIT can be useful for locating underground mineral deposits [77], detection of leaks in underground storage tanks [79] and for monitoring flows of injected fluids into the earth, for the purpose of oil extraction or environmental cleaning [80]. Finally, in nondestructive testing, EIT can be used for the detection of corrosion [81] and of small defects, such as cracks or voids, in metals $[5,6,24,37,45,83]$.

Electrical impedance tomography has been studied extensively in the last two decades and substantial progress has been made in the theoretical $[3,12,18,19,23,35,57,68,69,74,76,88,92]$, numerical $[17,18,22,27,33,34,40,50,63,64,66,82,86,94]$ and experimental $[11,27,54,71,79,87]$ aspects of the problem. Nevertheless, EIT remains an area of active research which continues to pose a variety of challenging questions to theoreticians, numerical analysts and experimentalists alike $[18,27,54,92]$.

In this paper, we propose a new reconstruction methodology for electrical impedance tomography, based on two, dual to each other, variational principles for the Neumann to Dirichlet and Dirichlet to Neumann maps, respectively. A variational, "equation-error" inversion method, based on the algorithm of Wexler, Fry and Neumann[93], has already been analyzed by Kohn and Vogelius [69] and implemented by Kohn and McKenney [66], where the electric current, the potential and the conductivity inside $\Omega$ are sought as minimizers of a functional which ensures that, at the "solution", Ohm's law is satisfied, at least for noiseless data. We consider here a different formulation which uses the variational principles as constraints on the set of admissible conductivity functions $\sigma$. Variational constraints have been proposed by Berryman and Kohn in [14] (see also [13, 15]). However, they have only been partially analyzed and their role in inversion has not been entirely understood. ${ }^{1}$ We give a detailed analysis of the variational constraints and we show how they can be used effectively in the numerical solution of the EIT problem. We propose a variety of reconstruction algorithms and we discuss some of their advantages and disadvantages. All these algorithms guarantee that, at least in the noiseless case, Ohm's law is satisfied inside $\Omega$ and, consequently, the measured boundary potential $V$ is fit in the natural, $H^{\frac{1}{2}}(\partial \Omega)$ norm. In theory, our numerical algorithms achieve the same objective as the variational method considered in $[66,69]$. However, due to the ill-posedness of the EIT problem, we note that, for noisy data, their numerical performance can be different. Finally, we point out the potential benefits of variationally constrained reconstructions over widely used imaging methods such as output least squares [18,27,94], where data is fit in the weaker, $L^{2}(\partial \Omega)$ norm, at the expense of loss in resolution of the images of $\sigma$.

This paper is organized as follows: In sections 2.1 and 2.2, we define the forward and inverse problem and we state the variational principles for the Neumann to Dirichlet and Dirichlet to Neumann maps. The variational constraints on $\sigma$, as defined originally by Berryman and Kohn [14], are given in section 2.3. In section 3, we motivate the use of variational constraints in the reconstruction algorithms. A detailed analysis of the variational constraints is given in section 4 . Based on this analysis, we propose a variety of reconstruction algorithms, in section 5.1. After a brief discussion of these algorithms, we concentrate on the constrained least squares formulation, which is implemented as discussed in 5.2. Numerical results and comparisons with traditional least squares and the variational algorithm in $[66,69]$ are given in section 6 . Finally, in section 7 , we give a summary of the paper and conclusions.

\footnotetext{
${ }^{1}$ There exists a variationally constrained numerical algorithm for the travel time tomography problem, due to Berryman [16]. However, this problem is considerably different than electrical impedance tomography, especially because Fermat's principle is not a minimum variational principle and, as such, it does not have a dual.
} 


\section{The Mathematical Model}

\subsection{The Forward Model}

We assume throughout the paper that the medium is isotropic. The electric potential and current density $\phi(\mathbf{x})$ and $\mathbf{j}(\mathbf{x})$, respectively, defined in terms of the electric and magnetic fields $\mathbf{E}(\mathbf{x})$ and $\mathbf{H}(\mathbf{x})$ as

$$
\mathbf{E}(\mathbf{x})=-\nabla \phi(\mathbf{x}), \quad \nabla \times \mathbf{H}(\mathbf{x})=\mathbf{j}(\mathbf{x})
$$

satisfy Ohm's law

$$
\mathbf{j}(\mathbf{x})=-\sigma(\mathbf{x}) \nabla \phi(\mathbf{x}),
$$

where $\sigma(\mathbf{x})$ is a scalar valued, strictly positive and bounded function in $\bar{\Omega}$, the closure of the domain. By definition, $\mathbf{j}$ is divergence free so Ohm's law (2.2) gives the partial differential equation

$$
\nabla \cdot[\sigma(\mathbf{x}) \nabla \phi(\mathbf{x})]=0 \text { in } \Omega,
$$

which we take with either Dirichlet boundary conditions

$$
\phi(\mathbf{x})=V(\mathbf{x}), \text { for } \mathbf{x} \in \partial \Omega
$$

or Neumann boundary conditions

$$
\sigma(\mathbf{x}) \nabla \phi(\mathbf{x}) \cdot \mathbf{n}(\mathbf{x}) \equiv \sigma(\mathbf{x}) \frac{\partial \phi(\mathbf{x})}{\partial n}=I(\mathbf{x}) \text { at } \partial \Omega, \text { such that } \int_{\partial \Omega} I(\mathbf{x}) d s(\mathbf{x})=0,
$$

where $\mathbf{n}(\mathbf{x})$ is the outer normal at $\mathbf{x} \in \partial \Omega$. It is well known that Dirichlet boundary value problem (2.3), (2.4), for arbitrary $V \in H^{\frac{1}{2}}(\partial \Omega)$, has a unique solution $\phi(\mathbf{x}) \in H^{1}(\Omega)$, at least in the weak sense [43]. Neumann boundary value problem $(2.3),(2.5)$, for $I \in H^{-\frac{1}{2}}(\partial \Omega)$, has a unique solution $\phi(\mathbf{x}) \in H^{1}(\Omega)$, up to an additive constant [43], which we fix by choosing the ground as

$$
\int_{\partial \Omega} \phi(\mathbf{x}) d s(\mathbf{x})=\int_{\partial \Omega} V(\mathbf{x}) d s(\mathbf{x})=0
$$

The electric current density satisfies equations

$$
\begin{aligned}
\nabla \times\left[\frac{1}{\sigma(\mathbf{x})} \mathbf{j}(\mathbf{x})\right] & =0 \text { and } \nabla \cdot \mathbf{j}(\mathbf{x})=0 \text { in } \Omega \\
-\mathbf{j}(\mathbf{x}) \cdot \mathbf{n}(\mathbf{x}) & =I(\mathbf{x}), \text { for } \mathbf{x} \in \partial \Omega, \text { such that } \int_{\partial \Omega} I(\mathbf{x}) d s(\mathbf{x})=0
\end{aligned}
$$

which, by (2.2), are equivalent to (2.3), (2.5). In particular, we have that (2.7) has a unique solution $\mathbf{j}(\mathbf{x})$, with bounded norm in $L^{2}(\Omega)$, which is related to potential $\phi(\mathbf{x}) \in H^{1}(\Omega)$, a solution of (2.3), (2.5), by Ohm's law (2.2).

Boundary value problems (2.3), (2.4); (2.3), (2.5) or, equivalently, (2.7), for a known function $\sigma(\mathbf{x})$ in $\Omega$ and data $I(\mathbf{x})$ or $V(\mathbf{x})$, given for all $\mathbf{x} \in \partial \Omega$, are referred to as forward mathematical models for electrical impedance tomography. 


\subsection{The Inverse Problem}

In EIT, the conductivity function $\sigma(\mathbf{x})$ is unknown and it is to be determined from simultaneous measurements of boundary voltages $V(\mathbf{x})$ and current densities $I(\mathbf{x})$, respectively. In this section, we define the Dirichlet to Neumann and Neumann to Dirichlet maps which relate $V(\mathbf{x})$ to $I(\mathbf{x})$. These maps depend nonlinearly on the unknown $\sigma(\mathbf{x})$ and they are data in inversion. We review some properties of these maps and we formulate the inverse problem.

The Dirichlet to Neumann $(\mathrm{DtN})$ map $\Lambda_{\sigma}: H^{\frac{1}{2}}(\partial \Omega) \rightarrow H^{-\frac{1}{2}}(\partial \Omega)$ is defined as

$$
\Lambda_{\sigma} V(\mathbf{x})=\sigma(\mathbf{x}) \frac{\partial \phi(\mathbf{x})}{\partial n} \text { for } \mathbf{x} \in \partial \Omega
$$

where $V(\mathbf{x})$ is arbitrary in $H^{\frac{1}{2}}(\partial \Omega)$ and $\phi(\mathbf{x})$ solves forward problem (2.3), (2.4). This map is selfadjoint and positive semidefinite with null space: $\mathcal{N}\left\{\Lambda_{\sigma}\right\}=\{V(\mathbf{x})=$ constant $\}$ (see for example $[18,27,89]$ and the references within). Moreover, it has the Dirichlet variational formulation [28]

$$
\left\langle V, \Lambda_{\sigma} V\right\rangle=\min _{\left.u\right|_{\partial \Omega}=V} \int_{\Omega} \sigma(\mathbf{x})|\nabla u(\mathbf{x})|^{2} d \mathbf{x}, \quad \text { for arbitrary } V(\mathbf{x}) \in H^{\frac{1}{2}}(\partial \Omega),
$$

where $\langle\cdot, \cdot\rangle$ denotes the $L_{2}(\partial \Omega)$ inner product

$$
\langle f, g\rangle=\int_{\partial \Omega} f(\mathbf{x}) g(\mathbf{x}) d s(\mathbf{x}) .
$$

The mathematical formulation of EIT, as first posed by Calderón [23], is: Find a bounded, strictly positive conductivity function $\sigma(\mathbf{x})$, given the DtN map $\Lambda_{\sigma}$. That this problem can be solved uniquely for a large class of functions $\sigma$ is established in $[35,36,67,74,88]$.

The generalized inverse of $\Lambda_{\sigma}$, the Neumann to Dirichlet (NtD) map $\left(\Lambda_{\sigma}\right)^{-1}: \mathcal{J} \rightarrow H^{\frac{1}{2}}(\partial \Omega)$, is defined on the restricted space of currents

$$
\mathcal{J}=\left\{I(\mathbf{x}) \in H^{-\frac{1}{2}}(\partial \Omega) \text { such that } \int_{\partial \Omega} I(\mathbf{x}) d s(\mathbf{x})=0\right\}
$$

and, for any $I(\mathbf{x}) \in \mathcal{J},\left(\Lambda_{\sigma}\right)^{-1} I(\mathbf{x})=\phi(\mathbf{x})$ at $\partial \Omega$, where $\phi(\mathbf{x})$ is the solution of Neumann boundary value problem $(2.3),(2.5),(2.6)$. The NtD map $\left(\Lambda_{\sigma}\right)^{-1}$ is self-adjoint and positive definite $[18,27$, 89], with Thomson variational formulation

$$
\left\langle I,\left(\Lambda_{\sigma}\right)^{-1} I\right\rangle=\min _{\substack{\nabla \cdot \mathbf{j}=0 \\-\mathbf{j} \cdot \mathbf{n} \mid \partial \Omega=I}} \int_{\Omega} \frac{1}{\sigma(\mathbf{x})}|\mathbf{j}(\mathbf{x})|^{2} d \mathbf{x}, \quad \text { for arbitrary } I(\mathbf{x}) \in \mathcal{J}
$$

(see for example [28]). That $\left(\Lambda_{\sigma}\right)^{-1}$ is the generalized inverse of $\Lambda_{\sigma}$, it can be seen from duality relations $[18,20,38]$

$$
\begin{aligned}
\left\langle V, \Lambda_{\sigma} V\right\rangle & =\sup _{I \in \mathcal{J}}\left\{2\langle I, V\rangle-\left\langle I,\left(\Lambda_{\sigma}\right)^{-1} I\right\rangle\right\}, \text { for any } V(\mathbf{x}) \in H^{\frac{1}{2}}(\partial \Omega), \\
\left\langle I,\left(\Lambda_{\sigma}\right)^{-1} I\right\rangle & =\sup _{V \in H^{\frac{1}{2}}(\partial \Omega)}\left\{2\langle I, V\rangle-\left\langle V, \Lambda_{\sigma} V\right\rangle\right\}, \text { for any } I(\mathbf{x}) \in \mathcal{J} .
\end{aligned}
$$

In practice, we do not have full knowledge of maps $\left(\Lambda_{\sigma}\right)^{-1}$ or $\Lambda_{\sigma}$. Instead, we have a set of $N$ experiments, where we define an excitation pattern $I_{e}(\mathbf{x}) \in \mathcal{J}$ and we measure the resulting 
voltage $V_{e}\left(\mathbf{x}_{p}\right)$, at discrete locations $\mathbf{x}_{p} \in \partial \Omega$ of the electrodes, along the boundary. Thus, the more realistic definition of EIT is: Find $\sigma$ from partial and usually noisy knowledge of the NtD map. A significant difficulty of the EIT problem is its severe ill-posedness which causes small perturbations of the boundary data to be exponentially amplified in the image of $\sigma$ inside $\Omega[4,7,18,31,32,55,85]$. Consequently, all reconstruction methods must be stabilized by some regularization approach, which ensures convergence by restricting $\sigma$ to a compact subset of $L^{\infty}(\Omega)$ (see for example $[25,40,41,51$, $72,90]$ or the statistical, Bayesian approaches in $[61-63,75])$.

\subsection{Variational Feasibility Constraints}

Variational constraints on the conductivity function $\sigma$ have been introduced by Berryman and Kohn [14], as follows:

Definition 1 We say that function $\sigma$ is Dirichlet feasible for boundary voltage $V_{e} \in H^{\frac{1}{2}}(\partial \Omega)$, if

$$
\left\langle V_{e}, \Lambda_{\sigma} V_{e}\right\rangle=\min _{\left.u\right|_{\partial \Omega}=V_{e}} \int_{\Omega} \sigma(\mathbf{x})|\nabla u(\mathbf{x})|^{2} d \mathbf{x} \geq\left\langle V_{e}, \Lambda_{\sigma^{\star}} V_{e}\right\rangle
$$

where $\sigma^{\star}$ is the true conductivity and $\left\langle V_{e}, \Lambda_{\sigma^{\star}} V_{e}\right\rangle=\int_{\partial \Omega} V_{e} I_{e} d s(\mathbf{x})=P_{e}$ is the measured power dissipated into heat. Moreover, we say that $\sigma$ is Dirichlet feasible, if (2.14) holds for all $V_{e} \in$ $H^{\frac{1}{2}}(\partial \Omega), e=1, \ldots N$.

The rationale behind this definition is given by variational principle (2.9), as follows: Take any $\phi \in H^{1}(\Omega)$, such that $\left.\phi\right|_{\partial \Omega}=V_{e}$ and obtain by $(2.9)$,

$$
\left\langle V_{e}, \Lambda_{\sigma^{\star}} V_{e}\right\rangle \leq \int_{\Omega} \sigma^{\star}(\mathbf{x})|\nabla \phi(\mathbf{x})|^{2} d \mathbf{x} .
$$

Now, let $\phi=\phi_{e}$, the solution of Dirichlet problem (2.3), (2.4) for conductivity $\sigma$, and suppose that $\sigma$ does not satisfy (2.14). Then,

$$
\left\langle V_{e}, \Lambda_{\sigma^{\star}} V_{e}\right\rangle>\left\langle V_{e}, \Lambda_{\sigma} V_{e}\right\rangle=\min _{\left.u\right|_{\partial \Omega}=V_{e}} \int_{\Omega} \sigma(\mathbf{x})|\nabla u(\mathbf{x})|^{2} d \mathbf{x}=\int_{\Omega} \sigma(\mathbf{x})\left|\nabla \phi_{e}(\mathbf{x})\right|^{2} d \mathbf{x}
$$

and $\sigma$ is deemed infeasible since it cannot be a solution according to (2.15).

Similar to Definition 1, we define the Thomson feasibility constraints as:

Definition 2 A function $\sigma$ is Thomson feasible for boundary electric current $I_{e} \in \mathcal{J}$, if

$$
\left\langle I_{e},\left(\Lambda_{\sigma}\right)^{-1} I_{e}\right\rangle=\min _{\substack{\nabla \cdot \mathbf{j}=0 \\-\left.\mathbf{j} \cdot \mathbf{n}\right|_{\partial \Omega}=I_{e}}} \int_{\Omega} \sigma^{-1}(\mathbf{x})|\mathbf{j}(\mathbf{x})|^{2} d \mathbf{x} \geq\left\langle I_{e},\left(\Lambda_{\sigma^{\star}}\right)^{-1} I_{e}\right\rangle,
$$

where $\left\langle I_{e},\left(\Lambda_{\sigma^{\star}}\right)^{-1} I_{e}\right\rangle=\int_{\partial \Omega} V_{e} I_{e} d s(\mathbf{x})=P_{e}$, the measured power dissipated into heat. Moreover, $\sigma$ is Thomson feasible, if (2.16) holds for all $I_{e} \in \mathcal{J}, e=1, \ldots N$.

Finally, we say that $\sigma$ is feasible if it is both Dirichlet and Thomson feasible. 


\section{Motivation for the Variational Formulation}

Let us define the set of admissible conductivity functions

$$
\mathcal{S}=\left\{\sigma(\mathbf{x}) \in L^{\infty}(\bar{\Omega}), \sigma(\mathbf{x}) \geq m\right\}
$$

where $m$ is some positive constant. Suppose that $\sigma^{\star} \in \mathcal{S}$ is the conductivity function to be imaged, such that, for a prescribed excitation current $I \in \mathcal{J}$, the boundary voltage is $V=\left(\Lambda_{\sigma^{\star}}\right)^{-1} I$. Ideally, we would like to image the conductivity by minimizing the operator norm

$$
\min _{\sigma \in \mathcal{S}}\left\|\left(\Lambda_{\sigma}\right)^{-1}-\left(\Lambda_{\sigma^{\star}}\right)^{-1}\right\|_{H^{-\frac{1}{2}}(\partial \Omega), H^{\frac{1}{2}}(\partial \Omega)},
$$

but, since only limited data is available, we can consider at best

$$
\min _{\sigma \in \mathcal{S}} \sum_{e=1}^{N}\left\|\left[\left(\Lambda_{\sigma}\right)^{-1}-\left(\Lambda_{\sigma^{\star}}\right)^{-1}\right] I_{e}\right\|_{H^{\frac{1}{2}}(\partial \Omega)}^{2}, \text { for } I_{e} \in \mathcal{J}, 1 \leq e \leq N,
$$

for some positive integer $N$. Then, one could attempt to solve nonlinear problem (3.3) with an iterative optimization algorithm, such as Newton's method [30]. However, due to the high cost of computing fractional order Sobolev space norms and the possible lack of differentiability of the functional in (3.3) [26, 32,33], no practical reconstruction algorithm uses formulation (3.3). Instead, one uses output least squares methods,

$$
\min _{\sigma \in \mathcal{S}} \sum_{e=1}^{N}\left\|\left[\left(\Lambda_{\sigma}\right)^{-1}-\left(\Lambda_{\sigma^{\star}}\right)^{-1}\right] I_{e}\right\|_{L^{2}(\partial \Omega)}^{2},
$$

where the objective function is an approximation of the Hilbert Schmidt norm of $\left(\Lambda_{\sigma}\right)^{-1}-\left(\Lambda_{\sigma^{\star}}\right)^{-1}$ over $L^{2}(\partial \Omega)[32]$ (the currents $I_{e}$ are scaled to unit norm). Obviously, formulation (3.4) can give at most a lower bound on (3.3) and, although numerically convenient, it could decrease the resolution of the image [26].

We propose to use the variational constraints, defined in Definitions 1 and 2, to achieve the minimization (3.3) in a computationally efficient manner. A possible approach is suggested by the following lemma:

Lemma 1 Let $I$ and $V$ be generic boundary data for imaging $\sigma^{\star}$ and let $\left\{\sigma_{k}(\mathbf{x})\right\}_{k \geq 1}$ be a sequence of functions in $\mathcal{S}$ such that,

$$
\lim _{k \rightarrow \infty}\left\langle I,\left(\Lambda_{\sigma_{k}}\right)^{-1} I\right\rangle=P \text { and } \lim _{k \rightarrow \infty}\left\langle V, \Lambda_{\sigma_{k}} V\right\rangle=P
$$

where $P=\langle I, V\rangle$. Then,

$$
\lim _{k \rightarrow \infty}\left\|\left(\Lambda_{\sigma_{k}}\right)^{-1} I-V\right\|_{H^{\frac{1}{2}(\partial \Omega)}}=0 .
$$

Proof: Let us denote by $\phi_{k}$ the potential which solves forward problem (2.3), (2.4), for conductivity $\sigma_{k}$ and Dirichlet data $V$. Let also $\mathbf{j}_{k}$ be the electric current density which solves problem (2.7) for conductivity $\sigma_{k}$ and Neumann data $I$. Equivalently, we have $\mathbf{j}_{k}=-\sigma_{k} \nabla \psi_{k}$, where $\psi_{k}$ solves Neumann boundary value problem (2.3), (2.5), (2.6). Then, integration by parts and assumption (3.5) give

$$
\left\langle I,\left(\Lambda_{\sigma_{k}}\right)^{-1} I\right\rangle+\left\langle V, \Lambda_{\sigma_{k}} V\right\rangle-2 P=\int_{\Omega} \sigma_{k}(\mathbf{x})\left|\nabla \phi_{k}(\mathbf{x})-\nabla \psi_{k}(\mathbf{x})\right|^{2} d \mathbf{x} \rightarrow 0, \text { as } k \rightarrow \infty .
$$


Moreover, by the coercivity of bilinear form $a(u, w)=\int_{\Omega} \sigma_{k} \nabla u \cdot \nabla w d \mathbf{x}$, for $u, w \in H^{1}(\Omega)$ satisfying $\int_{\partial \Omega} u d s=\int_{\partial \Omega} w d s=0$ [43], $\lim _{k \rightarrow \infty}\left\|\phi_{k}-\psi_{k}\right\|_{H^{1}(\Omega)}=0$ and, by the trace theorem [43], $\lim _{k \rightarrow \infty}\left\|V-\left(\Lambda_{\sigma_{k}}\right)^{-1} I\right\|_{H^{\frac{1}{2}(\partial \Omega)}}=0$.

Thus, we achieve the minimization (3.3), by seeking an inversion methodology which generates sequences $\left\{\sigma_{k}\right\}_{k>1}$ of functions in $\mathcal{S}$ which satisfy the feasibility constraints of Definitions 1 and 2 as equalities (or near equalities in the noisy case) in the limit $k \rightarrow \infty$. Such inversion methods are described in section 5.1 and they are based on the analysis of the variational constraints, which we give in section 4 . Note in particular that $\mathbf{j}_{k}$ and $\phi_{k}$ are related by Ohm's law inside $\Omega$ (except possibly for subsets of measure zero), since by (3.7), $\left\|\sigma_{k} \nabla \phi_{k}+\mathbf{j}_{k}\right\|_{L^{2}(\Omega)} \rightarrow 0$, as $k \rightarrow \infty$.

\section{Analysis of Variational Constraints}

\subsection{Theory}

As motivated by section 3, we wish to obtain, in a computationally efficient manner, a sequence $\left\{\sigma_{k}(\mathbf{x})\right\}_{k \geq 1}$ of conductivity functions satisfying (3.5). We begin by taking a closer look at output least squares methods, which generate sequences $\left\{\widetilde{\sigma}_{k}(\mathbf{x})\right\}_{k \geq 1}$ of conductivities such that

$$
\lim _{k \rightarrow \infty}\left\|\left(\Lambda_{\widetilde{\sigma}_{k}}\right)^{-1} I-V\right\|_{L^{2}(\partial \Omega)}=0
$$

Since

$$
\left\langle I,\left(\Lambda_{\widetilde{\sigma}_{k}}\right)^{-1} I\right\rangle-P=\int_{\partial \Omega} I\left[\left(\Lambda_{\widetilde{\sigma}_{k}}\right)^{-1} I-V\right] d s
$$

output least squares can give convergence of the Thomson constraint,

$$
\lim _{k \rightarrow \infty}\left\langle I,\left(\Lambda_{\widetilde{\sigma}_{k}}\right)^{-1} I\right\rangle=P
$$

provided that the excitation current $I$ is taken in $L^{2}(\partial \Omega)$. Now, for the Dirichlet constraint, we have

$$
\begin{aligned}
& \left\langle V, \Lambda_{\widetilde{\sigma}_{k}} V\right\rangle-P=\left\langle V, \Lambda_{\widetilde{\sigma}_{k}} V\right\rangle-\int_{\partial \Omega} V(\mathbf{x}) I(\mathbf{x}) d s(\mathbf{x}) \\
& =\left\langle V, \Lambda_{\widetilde{\sigma}_{k}} V\right\rangle-\int_{\partial \Omega} \widetilde{\phi}_{k}(\mathbf{x}) \widetilde{\sigma}_{k}(\mathbf{x}) \frac{\partial \widetilde{\psi}_{k}}{\partial n}(\mathbf{x}) d s(\mathbf{x}) \\
& =\left\langle V, \Lambda_{\widetilde{\sigma}_{k}} V\right\rangle-\int_{\Omega} \nabla \cdot\left[\widetilde{\sigma}_{k}(\mathbf{x}) \widetilde{\phi}_{k}(\mathbf{x}) \nabla \widetilde{\psi}_{k}(\mathbf{x})\right] d \mathbf{x} \\
& =\left\langle V, \Lambda_{\widetilde{\sigma}_{k}} V\right\rangle-\int_{\Omega} \nabla \cdot\left[\widetilde{\sigma}_{k}(\mathbf{x}) \widetilde{\psi}_{k}(\mathbf{x}) \nabla \widetilde{\phi}_{k}(\mathbf{x})\right] d \mathbf{x} \\
& =\int_{\partial \Omega}\left[V(\mathbf{x})-\left(\Lambda_{\widetilde{\sigma}_{k}}\right)^{-1} I\right] \Lambda_{\widetilde{\sigma}_{k}} V(\mathbf{x}) d s(\mathbf{x}),
\end{aligned}
$$

where $\widetilde{\phi}_{k}$ and $\widetilde{\psi}_{k}$ are the Dirichlet and Neumann potentials solving problems (2.3), (2.4) and (2.3), (2.5), respectively, for conductivity $\widetilde{\sigma}_{k}$ and data $V$ and $I$. However, since $\Lambda_{\widetilde{\sigma}_{k}} V \in H^{-\frac{1}{2}}(\partial \Omega)$, convergence of the Dirichlet constraint does not follow from (4.1).

To develop algorithms which give (3.5), we examine the relationship between the two feasibility constraints. We begin by showing that, for given data $I$ and $V$, the Thomson infeasible set is included in the Dirichlet feasible set (see Figure 1). Then, we could achieve convergence (3.5) of both constraints, to $P$, by confining all iterates $\sigma_{k}$ to the Dirichlet infeasible region and by letting the Thomson constraint converge to $P$. The latter can be achieved, for example, with output least squares, provided that we take $I$ sufficiently smooth (in $L^{2}(\partial \Omega)$ ) (see sections 3 and 5.1). 


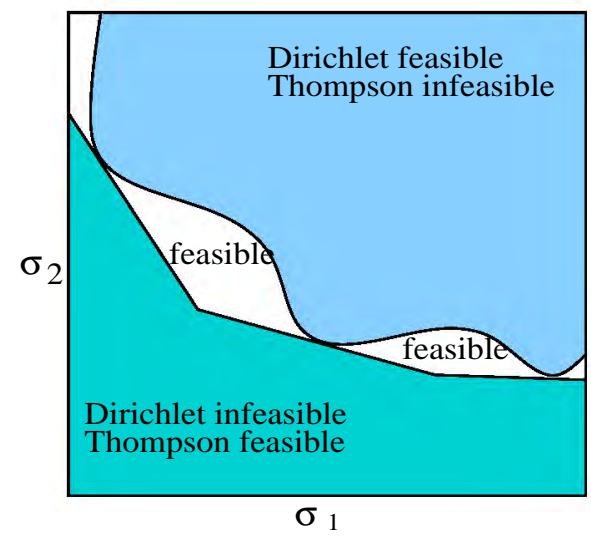

Figure 1: Take a planar section through the space of conductivity functions, where $\sigma$ is parameterized in terms of two values $\sigma_{1}$ and $\sigma_{2}$. The Thomson infeasibility region is contained in the Dirichlet feasible region, as stated by Lemma 2 .

Lemma 2 Let $\sigma \in \mathcal{S}$ be a conductivity function satisfying $\left\langle I,\left(\Lambda_{\sigma}\right)^{-1} I\right\rangle \leq P$, for some $I \in \mathcal{J}$ and $P=\langle I, V\rangle$. Then, $\left\langle V, \Lambda_{\sigma} V\right\rangle \geq P$.

Proof: The proof follows from duality relation (2.12). We have

$$
\left\langle V, \Lambda_{\sigma} V\right\rangle \geq 2\langle I, V\rangle-\left\langle I,\left(\Lambda_{\sigma}\right)^{-1} I\right\rangle \geq 2\langle I, V\rangle-P=P .
$$

Similarly, we obtain from duality relation (2.13) that the Dirichlet infeasible set in $\mathcal{S}$ is included in the Thomson feasible set, for data $I$ and $V$.

Lemma 3 Let $\sigma \in \mathcal{S}$ be a conductivity function satisfying $\left\langle V, \Lambda_{\sigma} V\right\rangle \leq P$, for some $V \in H^{\frac{1}{2}}(\partial \Omega)$ and $P=\langle I, V\rangle$. Then, $\left\langle I,\left(\Lambda_{\sigma}\right)^{-1} I\right\rangle \geq P$.

Note that, in general, given just one set of data $I, V$, the interiors of the Dirichlet and Thomson feasible sets are not disjoint. To guarantee that the two sets intersect just at the boundary, we need all measurements, as stated below.

Lemma 4 Let $\mathcal{D}$ and $\mathcal{T}$ be the interiors of the Dirichlet and Thomson feasibility sets, respectively,

$$
\begin{aligned}
& \mathcal{D}=\left\{\sigma \in \mathcal{S} \text { such that }\left\langle V, \Lambda_{\sigma} V\right\rangle>\left\langle V, \Lambda_{\sigma^{\star}} V\right\rangle, \text { for all } V \in H^{-\frac{1}{2}}(\partial \Omega)\right\}, \\
& \mathcal{T}=\left\{\sigma \in \mathcal{S} \text { such that }\left\langle I,\left(\Lambda_{\sigma}\right)^{-1} I\right\rangle>\left\langle I,\left(\Lambda_{\sigma^{\star}}\right)^{-1} I\right\rangle, \text { for all } I \in \mathcal{J}\right\} .
\end{aligned}
$$

Then, $\mathcal{D} \cap \mathcal{T}=\emptyset$.

Proof: Suppose that $\sigma \in \mathcal{T}$, such that

$$
2\langle I, V\rangle-\left\langle I,\left(\Lambda_{\sigma}\right)^{-1} I\right\rangle<2\langle I, V\rangle-\left\langle I,\left(\Lambda_{\sigma^{\star}}\right)^{-1} I\right\rangle,
$$

for all $I \in \mathcal{J}$ and arbitrary $V \in H^{-\frac{1}{2}}(\partial \Omega)$. Taking the sup over $I$ and using duality relation (2.12), we have

$$
\left\langle V, \Lambda_{\sigma} V\right\rangle=\sup _{I \in \mathcal{J}}\left\{2\langle I, V\rangle-\left\langle I,\left(\Lambda_{\sigma}\right)^{-1} I\right\rangle\right\} \leq \sup _{I \in \mathcal{J}}\left\{2\langle I, V\rangle-\left\langle I,\left(\Lambda_{\sigma^{\star}}\right)^{-1} I\right\rangle\right\}=\left\langle V, \Lambda_{\sigma^{\star}} V\right\rangle
$$


and so, $\sigma \notin \mathcal{D}$. In fact, if all measurements are available, by the uniqueness of solution of the inverse problem $^{2}$, the intersection of the feasibility sets consists of a single point $\sigma^{\star}$, the true solution.

When using a reconstruction algorithm which confines all iterates $\sigma_{k}$ inside (or outside) a feasibility set, one needs, for example, to find an appropriate initial guess. This can be easily done due to the monotonicity results:

Lemma 5 Let $\sigma, \widetilde{\sigma}$ be two functions in $\mathcal{S}$ and suppose that $\sigma(\mathbf{x}) \leq \widetilde{\sigma}(\mathbf{x})$, for all $\mathbf{x}$ in $\Omega$, with the possible exception of subsets of measure zero. Then,

$$
\left\langle V, \Lambda_{\sigma} V\right\rangle \leq\left\langle V, \Lambda_{\tilde{\sigma}} V\right\rangle \text { and }\left\langle I,\left(\Lambda_{\sigma}\right)^{-1} I\right\rangle \geq\left\langle I,\left(\Lambda_{\tilde{\sigma}}\right)^{-1} I\right\rangle,
$$

for any $V \in H^{\frac{1}{2}}(\partial \Omega)$ and $I \in \mathcal{J}$.

Proof: The proof is given by Berryman in [15]. We repeat it here, for completeness. From variational principle $(2.9)$, we have

$$
\begin{aligned}
\left\langle V, \Lambda_{\sigma} V\right\rangle & =\min _{\left.u\right|_{\partial \Omega}=V} \int_{\Omega} \sigma(\mathbf{x})|\nabla u(\mathbf{x})|^{2} d \mathbf{x} \leq \int_{\Omega} \sigma(\mathbf{x})|\nabla \widetilde{\phi}(\mathbf{x})|^{2} d \mathbf{x} \leq \int_{\Omega} \widetilde{\sigma}(\mathbf{x})|\nabla \widetilde{\phi}(\mathbf{x})|^{2} d \mathbf{x} \\
& =\min _{\left.u\right|_{\partial \Omega}=V} \int_{\Omega} \widetilde{\sigma}(\mathbf{x})|\nabla u(\mathbf{x})|^{2} d \mathbf{x}=\left\langle V, \Lambda_{\tilde{\sigma}} V\right\rangle .
\end{aligned}
$$

Similarly, from (2.11), we have

$$
\begin{aligned}
\left\langle I,\left(\Lambda_{\left.\tilde{\sigma})^{-1} I\right\rangle=}\right.\right. & \min _{\substack{\nabla \cdot \mathbf{i}=0 \\
-\mathbf{i} \cdot \mathbf{n} \mid \partial \Omega=I}} \int_{\Omega} \frac{1}{\widetilde{\sigma}(\mathbf{x})}|\mathbf{i}(\mathbf{x})|^{2} d \mathbf{x} \\
= & \int_{\Omega} \frac{1}{\widetilde{\sigma}(\mathbf{x})}|\widetilde{\mathbf{j}}(\mathbf{x})|^{2} d \mathbf{x} \leq \int_{\Omega} \frac{1}{\widetilde{\sigma}(\mathbf{x})}|\mathbf{j}(\mathbf{x})|^{2} d \mathbf{x} \leq \int_{\Omega} \frac{1}{\sigma(\mathbf{x})}|\mathbf{j}(\mathbf{x})|^{2} d \mathbf{x} \\
= & \min _{\substack{\nabla \cdot \mathbf{i}=0\\
}} \int_{\Omega} \frac{1}{\sigma(\mathbf{x})}|\mathbf{i}(\mathbf{i})|^{2} d \mathbf{x}=\left\langle I,\left(\Lambda_{\sigma}\right)^{-1} I\right\rangle .
\end{aligned}
$$

We end this section with the well known convexity result (see for example $[15,59]$ ):

Lemma 6 Let $\sigma, \widetilde{\sigma}$ be two functions belonging to the Dirichlet feasible set for data $I$ and $V$. Then, linear combination $\lambda \sigma+(1-\lambda) \widetilde{\sigma}$, for $0 \leq \lambda \leq 1$, is Dirichlet feasible, as well.

Note however that the set of Thomson feasible conductivities is not convex.

\subsection{Examples}

We give a few examples of the feasibility regions analyzed in section 4.1, for a two-dimensional conductivity $\sigma(x, y)$ in a unit square $\Omega$. We take $\sigma=1$ everywhere in the domain, except for two rectangular inclusions, where $\sigma$ equals $\sigma_{1}$ or $\sigma_{2}$. By varying $\sigma_{1}$ and $\sigma_{2}$, we obtain, similar to Figure 1 , a planar section through the space of conductivity functions. We calculate the Dirichlet and Thomson feasibility regions, for various data $I, V$ and different locations of the inhomogeneities inside $\Omega$.

\footnotetext{
${ }^{2}$ Uniqueness has been proven for a large class of conductivities in [21,35, 36, 67, 74, 76, 88]. All these results require some smoothness assumptions on $\sigma$ but the result may hold for the entire set $\mathcal{S}$.
} 

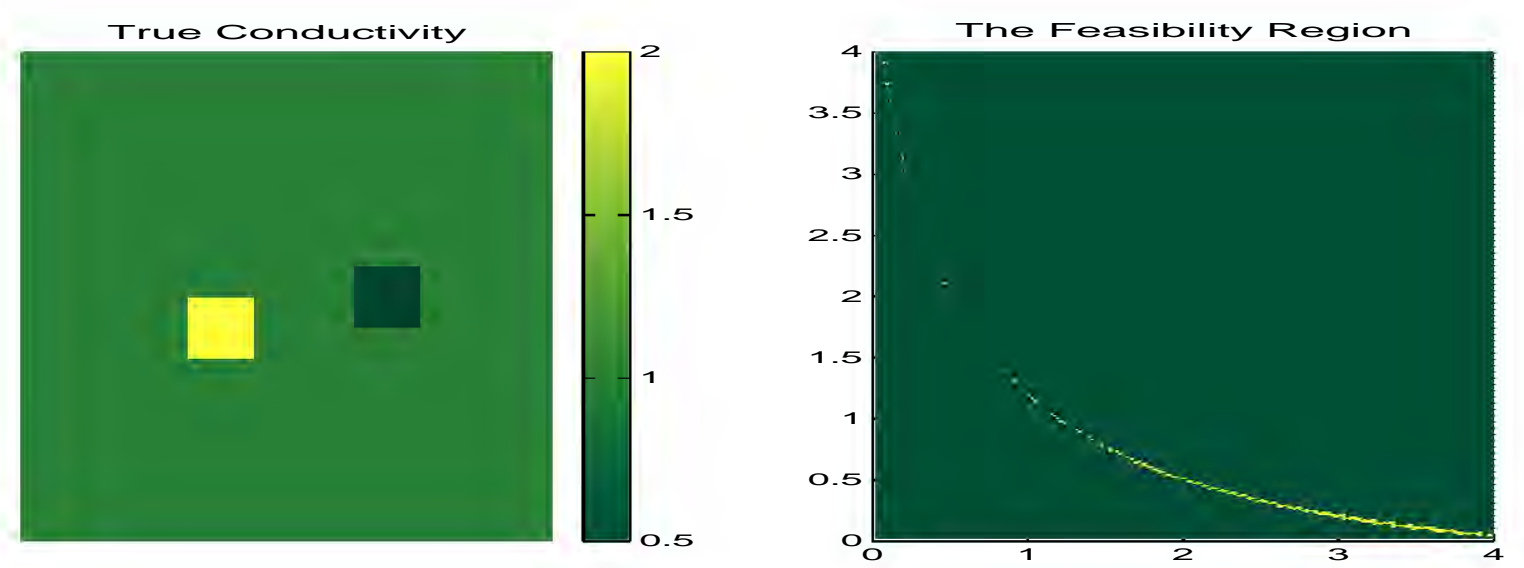

Figure 2: On the left, we plot $\sigma$ as a function of $x$ and $y$. On the right, we show in yellow the feasibility region in the plane parameterized by $\sigma_{1}$ and $\sigma_{2}$. The conductivity distribution on the left is not easily distinguishable by the experiment $I, V$, because the boundaries of the two feasibility regions intersect along a long arc in the plane.

Let us inject unit current near the lower left corner of $\Omega$ and take it out near its right upper corner. For such a fixed $I$, we study the effect of the location of the inclusions on the feasibility regions. In Figure 2, we have two inclusions, of conductivity $\sigma_{1}=2$ and $\sigma_{2}=0.5$, near the center of the domain. We show in the right picture the feasibility region (the intersection of the Dirichlet and Thomson feasibility regions), for data $I, V$. Note that the feasibility region collapses to a long arc in the part of the plane displayed in the figure. Any pair $\left(\sigma_{1}, \sigma_{2}\right)$ along this arc gives an almost perfect fit of the data and so, the conductivity distribution in Figure 2 cannot be determined by just this experiment $I, V$. However, as shown in Figure 3, inclusions which are close to the boundary are easily distinguished, because the boundaries of the Dirichlet and Thomson feasibility regions intersect at one point, the exact conductivity.

Next, we fix the conductivity distribution to that shown in Figure 4, where we have two inclusions of conductivity $\sigma_{1}=2$ and $\sigma_{2}=0.5$ in a background of conductivity 1 , and we study the effect of the excitation current on the feasibility set. In the middle picture of Figure 4, we take the optimal current which distinguishes best the inclusions in $\Omega[26,55]$, whereas in the right picture of Figure 4, we inject and take out unit current near the upper left and lower right corners of $\Omega$, respectively. As seen from Figure 4, the latter is a bad excitation, which cannot distinguish the conductivity, since the feasibility region collapses to a long arc over a large region in the plane $\left(\sigma_{1}, \sigma_{2}\right)$.

Clearly, for more general conductivity distributions, it is typically not possible to find $\sigma$ with just one experiment and so, more data is needed and one should look at the intersection of the feasibility regions for all available current excitations. This could be useful, in particular, in special cases where the conductivity is known a priori to be piecewise constant over $M$ given subdomains (i.e. $\sigma$ is parameterized in terms of $\left.\sigma_{1}, \sigma_{2}, \ldots \sigma_{M}\right)^{3}$, because we could use the feasibility constraints to determine the error in the recovered $\sigma_{j}$, for $j=1, \ldots M$.

\footnotetext{
${ }^{3}$ Such problems may arise in medical imaging, where the borders of the subdomains of constant conductivity could be determined from CAT scans [60].
} 

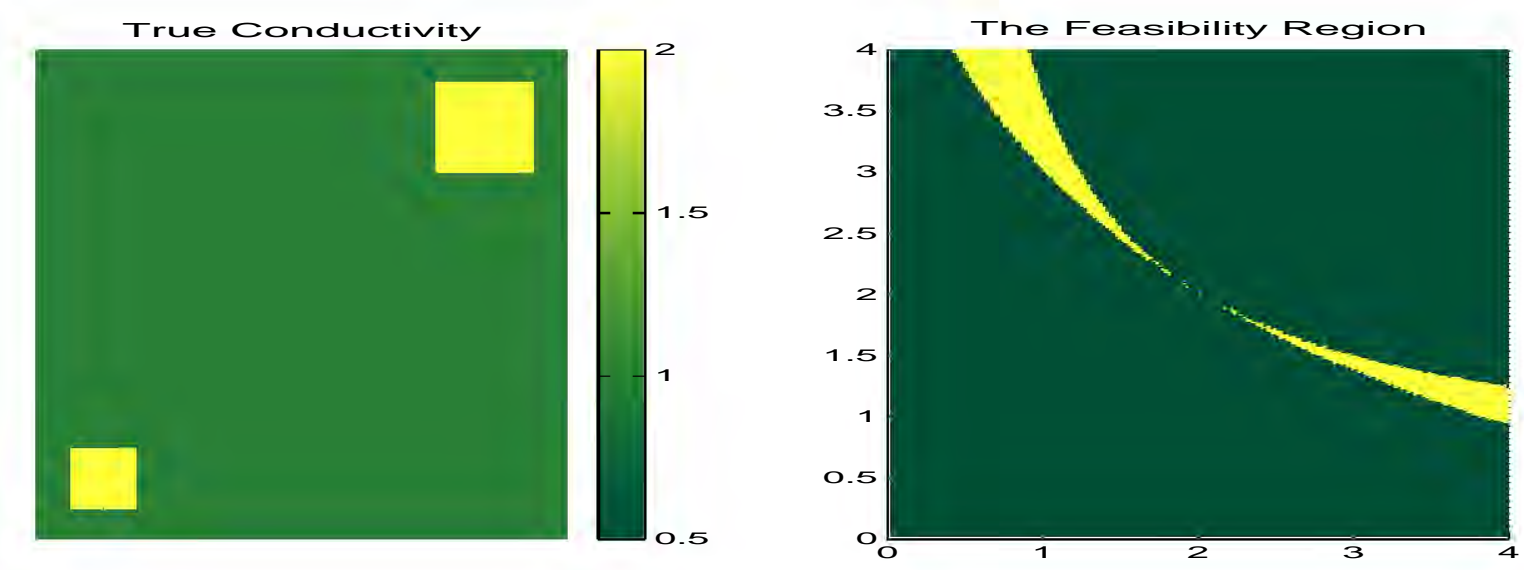

Figure 3: The conductivity distribution on the left is easily distinguishable by the experiment $I, V$, because the boundaries of the two feasibility regions intersect just at one point, where the true solution lies.
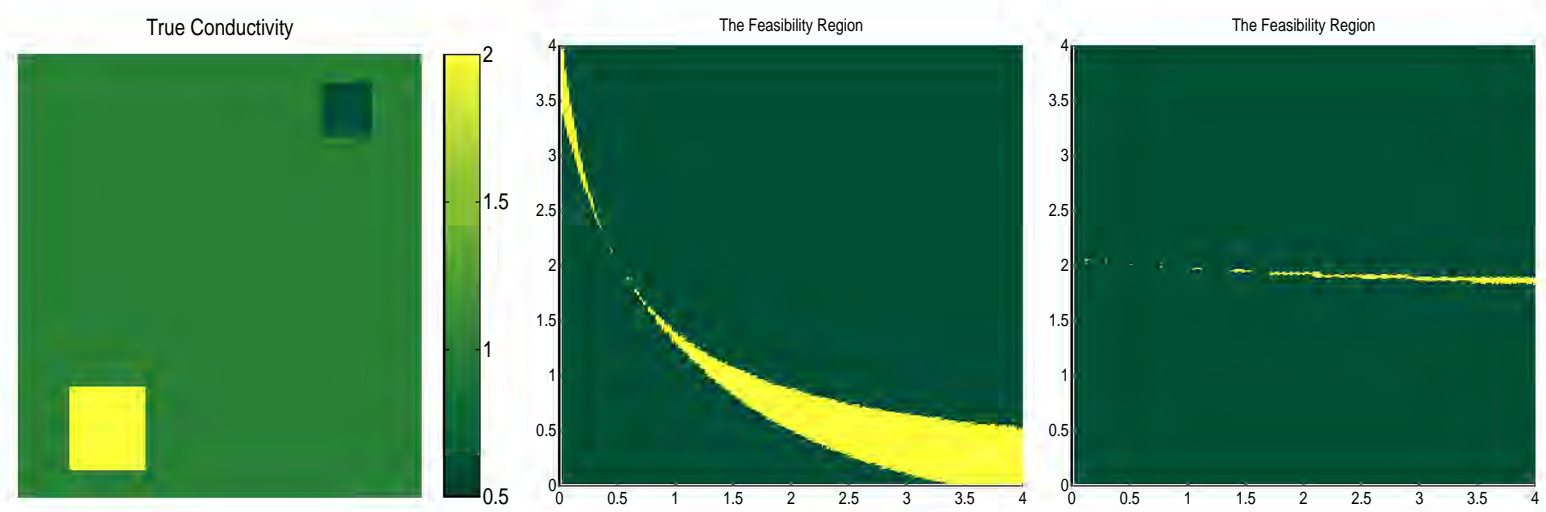

Figure 4: The conductivity distribution in the left picture can be easily distinguished with the optimal current excitation, but it cannot be distinguished by a bad example of a current excitation.

\section{Variationally Constrained Reconstruction Algorithms}

In this section, we introduce several variational algorithmic approaches for the numerical solution of the EIT problem. Then, we describe in detail our implementation of two variational algorithms and the unconstrained output least squares algorithm.

\subsection{Several Algorithmic Approaches}

We assume that $N$ experiments have been conducted with boundary excitation currents $I_{e} \in \mathcal{J}$, that the corresponding voltages $V_{e}$ at $\partial \Omega$ have been measured ${ }^{4}$ and that the power dissipated into heat, $P_{e}=\int_{\partial \Omega} I_{e} V_{e} d s$, has been estimated, for $e=1,2, \cdots, N$.

\footnotetext{
${ }^{4}$ Note that, in all our reconstructions, $I_{e}$ and $V_{e}$ are given at discrete locations along $\partial \Omega$. For the numerical solution of the forward problems and for the calculation of $P_{e}$, we take piecewise linear interpolation of the data, at the boundary, between the points of "measurement".
} 


\subsubsection{Constrained Least Squares Approach}

As is shown in section 3 , in the output least squares formulation, for $I_{e} \in \mathcal{J} \cap L^{2}(\partial \Omega)$, the convergence of objective function (3.4) to zero implies the convergence of the Thomson constraint, to $P_{e}$, but not necessarily that of the Dirichlet constraint. However, in light of the results in section 4.1, we can achieve the desired convergence (3.5) of both Dirichlet and Thomson constraints on $\sigma$, by the constrained least squares approach:

$$
\min _{\sigma \in \mathcal{S}} \sum_{e=1}^{N}\left\|\left(\Lambda_{\sigma}\right)^{-1} I_{e}-V_{e}\right\|_{L^{2}(\partial \Omega)}^{2} \quad \text { s.t. }\left\langle V_{e}, \Lambda_{\sigma} V_{e}\right\rangle \leq P_{e}, \quad e=1, \ldots, N,
$$

where $I_{e} \in \mathcal{J} \cap L^{2}(\partial \Omega)$ and $V_{e}=\left(\Lambda_{\sigma^{\star}}\right)^{-1} I_{e} \in H^{\frac{1}{2}}(\partial \Omega)$. By requiring $\sigma$ to be in the Dirichlet infeasibility region, we ensure that, at the minimizer, both Dirichlet and Thomson constraints are satisfied as equalities (or near equalities in the presence of noise). Equivalently, a minimizer of (5.1) lies at the intersection between the Dirichlet and Thomson feasibility boundaries.

\subsubsection{Equation Error Approach}

If follows from the proof of Lemma 1 that

$$
\left\langle V_{e}, \Lambda_{\sigma} V_{e}\right\rangle+\left\langle I_{e},\left(\Lambda_{\sigma}\right)^{-1} I_{e}\right\rangle-2 P_{e}=\int_{\Omega} \sigma(\mathbf{x})\left|\nabla\left[\psi_{e}(\mathbf{x})-\phi_{e}(\mathbf{x})\right]\right|^{2} d \mathbf{x} \geq 0
$$

where $\phi_{e}$ is the solution of Dirichlet problem (2.3) and (2.4), with data $V_{e}=\left(\Lambda_{\sigma^{\star}}\right)^{-1} I_{e} \in H^{\frac{1}{2}}(\partial \Omega)$, and $\psi_{e}$ is the solution of Neumann problem (2.3) and (2.5), with data $I_{e} \in \mathcal{J}$. Then, a global minimizer of

$$
\min _{\sigma \in \mathcal{S}} \sum_{e=1}^{N}\left(\left\langle V_{e}, \Lambda_{\sigma} V_{e}\right\rangle+\left\langle I_{e},\left(\Lambda_{\sigma}\right)^{-1} I_{e}\right\rangle-2 P_{e}\right)
$$

is a solution of the EIT problem, at which Ohm's law $\mathbf{j}_{e}=-\sigma \nabla \psi_{e}=-\sigma \nabla \phi_{e}$ is satisfied (in $L^{2}(\Omega)$ sense), at least for noiseless data. We note that this approach is equivalent to that of $[66,69,93]$ and, as such, we refer to it as the equation-error variational formulation.

\subsubsection{Other Approaches}

Since the Dirichlet infeasible region is a subset of the Thomson feasible region (see section 4.1), the minimization of Thomson functional $\left\langle I_{e},\left(\Lambda_{\sigma}\right)^{-1} I_{e}\right\rangle-P_{e} \geq 0$, over Dirichlet infeasible $\sigma$, ensures the desired convergence (3.5). Explicitly, we take:

$$
\min _{\sigma \in \mathcal{S}} \sum_{e=1}^{N}\left(\left\langle I_{e},\left(\Lambda_{\sigma}\right)^{-1} I_{e}\right\rangle-P_{e}\right) \quad \text { s.t. }\left\langle V_{e}, \Lambda_{\sigma} V_{e}\right\rangle \leq P_{e}, \quad e=1, \ldots, N,
$$

for $I_{e} \in \mathcal{J}$ and $V_{e}=\left(\Lambda_{\sigma^{*}}\right)^{-1} I_{e} \in H^{\frac{1}{2}}(\partial \Omega)$. Similarly, we can have a "dual" formulation, where we constrain $\sigma$ to the Thomson infeasibility region and we minimize the Dirichlet functional $\left\langle V_{e}, \Lambda_{\sigma} V_{e}\right\rangle-P_{e} \geq 0$ :

$$
\min _{\sigma \in \mathcal{S}} \sum_{e=1}^{N}\left(\left\langle V_{e}, \Lambda_{\sigma} V_{e}\right\rangle-P_{e}\right) \quad \text { s.t. }\left\langle I_{e},\left(\Lambda_{\sigma}\right)^{-1} I_{e}\right\rangle \leq P_{e}, \quad e=1, \ldots, N .
$$


In addition, there exist other possibilities. For example, one may directly seek to find a conductivity in the intersection of the Thomson and Dirichlet feasibility boundaries by minimizing the distance between the two infeasibility regions:

$$
\min _{\sigma_{D}, \sigma_{T}}\left\|\sigma_{D}-\sigma_{T}\right\|_{L^{2}(\Omega)}^{2} \quad \text { s.t. }\left\langle V_{e}, \Lambda_{\sigma_{D}} V_{e}\right\rangle \leq P_{e}, \quad\left\langle I_{e},\left(\Lambda_{\sigma_{T}}\right)^{-1} I_{e}\right\rangle \leq P_{e}, \quad e=1, \ldots, N .
$$

Here, $\sigma_{D}$ is in the Dirichlet infeasibility region and $\sigma_{T}$ in the Thompson infeasibility region. The reconstruction can then be given by the pointwise average of $\sigma_{D}$ and $\sigma_{T}$.

Although the formulations based on variational constraints are "equivalent" in a mathematical sense, their practical performance may differ from case to case. Our numerical experiments suggest that approaches (5.1), (5.4) or (5.5) perform similarly in the tested cases, and that they are the most consistent ones of all the formulations that we have considered. The equation-error approach (5.3) performs equally well for noiseless data but it can behave differently for noisy measurements. Finally, approach (5.6) is the least successful one. Our preliminary studies suggest that the objective function in (5.6) has many local minima and, as such, the iterative optimization process stagnates after just a few steps, for most initial guesses. Due to this behavior, we have have not been able to obtain reasonable reconstructions with formulation (5.6), at least for realistic initial starting values of $\sigma$.

\subsection{Implementation}

We now describe our implementation of three formulations: (1) the constrained least squares approach, (2) the equation-error approach and (3) the unconstrained output least squares approach. We choose the constrained least squares approach to represent the newly proposed variationally constrained formulations, and we compare it with the other two approaches.

\subsubsection{The optimization method}

In an attempt to achieve better scaling, we have implemented normalized versions of the three formulation; namely, the normalized constrained least squares formulation:

$$
\min _{\sigma \in \mathcal{S}} \sum_{e=1}^{N} \frac{\left\|\left(\Lambda_{\sigma}\right)^{-1} I_{e}-V_{e}\right\|_{L^{2}(\partial \Omega)}^{2}}{\left\|V_{e}\right\|_{L^{2}(\partial \Omega)}^{2}} \quad \text { s.t. }\left\langle V_{e}, \Lambda_{\sigma} V_{e}\right\rangle \leq P_{e}, \quad e=1, \ldots, N,
$$

the normalized equation-error formulation:

$$
\min _{\sigma \in \mathcal{S}} \sum_{e=1}^{N} \frac{\left\langle V_{e}, \Lambda_{\sigma} V_{e}\right\rangle+\left\langle I_{e},\left(\Lambda_{\sigma}\right)^{-1} I_{e}\right\rangle-2 P_{e}}{2 P_{e}},
$$

and the normalized unconstrained output least squares formulation:

$$
\min _{\sigma \in \mathcal{S}} \sum_{e=1}^{N} \frac{\left\|\left(\Lambda_{\sigma}\right)^{-1} I_{e}-V_{e}\right\|_{L^{2}(\partial \Omega)}^{2}}{\left\|V_{e}\right\|_{L^{2}(\partial \Omega)}^{2}}
$$

To solve the constrained least squares problem (5.7), we approximately solve a sequence of unconstrained logarithmic barrier subproblems:

$$
\min _{\sigma \in \mathcal{S}} \sum_{e=1}^{N}\left(\frac{\left\|\left(\Lambda_{\sigma}\right)^{-1} I_{e}-V_{e}\right\|_{L^{2}(\partial \Omega)}^{2}}{\left\|V_{e}\right\|_{L^{2}(\partial \Omega)}^{2}}-\mu \log \left(P_{e}-\left\langle V_{e}, \Lambda_{\sigma} V_{e}\right\rangle\right)\right),
$$


corresponding to a sequence of positive and decreasing parameter values for $\mu$. In this so-called log-barrier approach, all iterates are required to be strictly feasible with respect to the constraints in (5.7). It is known [42] that under suitable conditions,

$$
\lim _{\mu \rightarrow 0} \sigma(\mu)=\sigma^{*}
$$

where $\sigma(\mu)$ solves (5.10) for given $\mu>0$ and $\sigma^{*}$ is a solution to (5.7). In our implementation, we start with the initial value $\mu=10^{-4}$ and every time decrease $\mu$ by a factor of 2 . The criterion that triggers a reduction in $\mu$ is that the relative change of the objective value in two consecutive iterations is less than $10^{-5}$ or that a maximum number of 1000 iterations has been reached, whichever comes first.

To solve the unconstrained optimization problems (5.8), (5.9) and (5.10), we apply the steepest descent (or gradient) method along with a back-tracking line search to ensure that the Armijo condition for sufficient decrease in the objective value (see page 118 in [30], for example) is satisfied at every iteration. The back-tracking line search begins with a step size of $\lambda=1$ and decreases $\lambda$ by a factor of 2 until the sufficient decrease condition has been met or until $\lambda \leq 2^{-10}$.

The steepest descent method is simple to implement and reliable in terms of convergence. Besides its simplicity, with an appropriate step control mechanism the steepest descent method seems to have the benefit of providing an iterative regularization effect for ill-posed problems. Although the method requires a large number of iterations to achieve a relatively high accuracy, it is sufficient for our purpose of evaluating the performance of the formulations in terms of reconstruction quality.

For each algorithm, we stop the iterations whenever the relative change in the objective function is less than $10^{-16}$, or a maximum number of iterations of 10000 has been reached. These stringent stopping criteria enable us to observe the asymptotic behavior of the algorithms. Indeed, in one of the experiments we were able to observe (see Figure 7) that the output least squares method eventually starts to increase the relative error in conductivity after more than two thousand iterations of decrease, while the other two methods continue to decrease the error all the way to the end.

\subsubsection{Discretization and calculation of derivatives}

In our implementation, we only consider the two dimensional problem with the domain $\Omega$ being the unit square. This simple model allows a simple implementation and manageable computational times, while being adequate for the purpose of evaluating the strength and weakness of different formulations. For convenience, we assume that the conductivity is known at the boundary of the domain. In all simulations included in this paper, we discretize the conductivity in terms of its nodal values, as a piecewise linear function, on a $16 \times 16$ uniform mesh of $\Omega$. On the boundary, $\sigma$ is set to be the constant one (1.0) at each of the 64 boundary nodes. The unknowns are the values of the conductivity at each of the 225 interior nodes. We use the first $N$ optimal current excitation vectors as described in $[27,46,55]$. In our experiments, we set $N=15, N=18$, and $N=19$ for test conductivity models 1,2 , and 3 , illustrated in figure 5, respectively. For each experiment, we solve the Dirichlet and Neumann problems for the current conductivity, at any iterate $\sigma$, on a $32 \times 32$ uniform triangularization of the domain $\Omega$ using the piecewise linear, finite elements method.

The quadrature rules for the approximation of the constraints are defined such that the important identity (5.2) is preserved as much as possible in the discrete setting. Let us denote by $\bar{G}=G \cup \partial G$ the grid for the numerical solution of the forward problems, where $G$ is the set of 
interior grid points and $\partial G$ is the set of boundary points. The Neumann potential $\psi$ is given by:

$$
\psi(\mathbf{x})=\sum_{j \in \bar{G}} \psi_{j} b_{j}(\mathbf{x})
$$

where $b_{j}(\mathbf{x})$ are the usual piecewise linear basis functions for the uniform triangulation on $\bar{G}$ and $\psi_{j}$ are the unknown values of $\psi$ at the grid points $j \in \bar{G}$, calculated such that

$$
\int_{\Omega} \sigma(\mathbf{x}) \nabla v(\mathbf{x}) \cdot \nabla \psi(\mathbf{x}) d \mathbf{x}=\int_{\partial \Omega} I(\mathbf{x}) v(\mathbf{x}) d s(\mathbf{x}), \text { for all piecewise linear functions } v(\mathbf{x}) .
$$

Then, by setting $v(\mathbf{x})=\psi(\mathbf{x})$ in (5.12), we obtain for the Thomson constraint

$$
\left\langle I,\left(\Lambda_{\sigma}\right)^{-1} I\right\rangle \approx \sum_{j \in \partial G} \psi_{j} \int_{\partial \Omega} I(\mathbf{x}) b_{j}(\mathbf{x}) d s(\mathbf{x})=\int_{\Omega} \sigma(\mathbf{x})|\nabla \psi(\mathbf{x})|^{2} d \mathbf{x} .
$$

The Dirichlet potential $\phi$ is given by

$$
\phi(\mathbf{x})=\sum_{j \in \partial G} V_{j} b_{j}(\mathbf{x})+\sum_{j \in G} \phi_{j} b_{j}(\mathbf{x})
$$

where $V_{j}$ is the given voltage at $j \in \partial G$ and where $\phi_{j}$, for $j \in G$, are calculated such that

$$
\int_{\Omega} \sigma(\mathbf{x}) \nabla v(\mathbf{x}) \cdot \nabla \phi(\mathbf{x}) d \mathbf{x}=0, \text { for all piecewise linear functions } v(\mathbf{x}) \text { vanishing at } \partial G .
$$

Taking $v(\mathbf{x})=\phi(\mathbf{x})$ in $(5.12)$, we have

$$
P=\langle I, V\rangle \approx \sum_{j \in \partial G} V_{j} \int_{\partial \Omega} I(\mathbf{x}) b_{j}(\mathbf{x}) d s(\mathbf{x})=\int_{\Omega} \sigma(\mathbf{x}) \nabla \psi(\mathbf{x}) \cdot \nabla \phi(\mathbf{x}) d \mathbf{x} .
$$

Finally, from (5.15), taking $v(\mathbf{x})=\phi(\mathbf{x})-\sum_{j \in \partial G} V_{j} b_{j}(\mathbf{x})$, we have

$$
\left\langle V, \Lambda_{\sigma} V\right\rangle \approx \sum_{j \in \partial G} V_{j} \int_{\Omega} \sigma(\mathbf{x}) \nabla b_{j}(\mathbf{x}) \cdot \nabla \phi(\mathbf{x}) d \mathbf{x}=\int_{\Omega} \sigma(\mathbf{x})|\nabla \phi(\mathbf{x})|^{2} d \mathbf{x}
$$

where the integration in the left hand side is limited to the triangles with at least one node in $\partial G$.

We note that formulations (5.8) and (5.10) require the solutions of both Dirichlet and Neumann problems, while for unconstrained output least squares (5.9), only the Neumann problems need to be solved. Nevertheless, the stiffness matrices $A_{D}$ and $A_{N}$ that one inverts in the numerical solution of Dirichlet and Neumann problems (5.15) and (5.12), respectively, are closely related to each other. In fact, $A_{D}$ is a subblock of $A_{N}$ and this can be used towards reducing the computational cost ${ }^{5}$.

An important computational task in our implementation is the evaluation of the first derivatives of the objective functionals. An efficient computation of the derivatives is given by the adjoint method (see for example [33]). Straightforward calculations [48] give that the first derivative of $\left\langle V, \Lambda_{\sigma} V\right\rangle$, with respect to $\sigma$, is given, pointwise for $\mathbf{x} \in \Omega$, by

$$
\left(\mathcal{D}\left\langle V, \Lambda_{\sigma} V\right\rangle\right)(\mathbf{x})=|\nabla \phi(\mathbf{x})|^{2}
$$

\footnotetext{
${ }^{5}$ For example, one can use a block factorization approach where, once $A_{D}$ has been factorized, $A_{N}$ can be factorized at a small extra cost (see [47], for example).
} 
and it is thus based solely on the previously computed approximation of $\phi$, the solution of the Dirichlet problem for conductivity $\sigma$. The derivative of $\left\langle I,\left(\Lambda_{\sigma}\right)^{-1} I\right\rangle$, with respect to $\sigma$, is given, pointwise for $\mathbf{x} \in \Omega$, by [48]

$$
\left(\mathcal{D}\left\langle I,\left(\Lambda_{\sigma}\right)^{-1} I\right\rangle\right)(\mathbf{x})=-|\nabla \psi(\mathbf{x})|^{2},
$$

where $\psi$ is the Neumann potential. Finally, the derivative of the output least squares functional is

$$
\left(\mathcal{D}\left\|\left(\Lambda_{\sigma}\right)^{-1} I-V\right\|_{L^{2}(\partial \Omega)}^{2}\right)(\mathbf{x})=-2 \nabla \psi(\mathbf{x}) \cdot \nabla \tau(\mathbf{x}),
$$

where $\tau$ is the (piecewise linear) adjoint potential satisfying

$$
\int_{\Omega} \sigma(\mathbf{x}) \nabla v(\mathbf{x}) \cdot \nabla \tau(\mathbf{x}) d \mathbf{x}=\int_{\partial \Omega}\left[\left(\Lambda_{\sigma}\right)^{-1} I(\mathbf{x})-V(\mathbf{x})\right] v(\mathbf{x}) d s(\mathbf{x})
$$

for all piecewise linear functions $v(\mathbf{x})$. The calculation of $\tau$ and $\psi$ require the inversion of the same stiffness matrix $A_{N}$, so (5.20) is calculated with little extra computation.

All three algorithms were implemented in Fortran 90. We use the software package SuperLU $[8,29]$ to efficiently solve the sparse linear systems resulting from the finite-element discretization.

\section{$6 \quad$ Numerical Results}

In this section, we present numerical results and we compare the performance of the three implemented algorithms (see section 5.2). We stress that the objective of our numerical comparison is to evaluate the reconstruction quality of these algorithms. Therefore, we will not include computational time in the presented results. Although our current implementations are adequately efficient for all three algorithms, there certainly exists ample room for further improvements.

To make a fair comparison, we use the same simulated data for all three methods, and we start each algorithm from the same initial guess, conductivity $\sigma^{0} \equiv 1$ at all nodes. However, for the constrained least squares formulation, such an initial guess may not be feasible with respect to the constraints $\left\langle V_{e}, \Lambda_{\sigma} V_{e}\right\rangle \leq P_{e}$ for $e=1,2, \cdots, N$. In this case, invoking the monotonicity of the Dirichlet constraint (Lemma 5), we decrease the value of $\sigma^{0}$ at all the interior nodes of $\Omega$ until the constraints are strictly satisfied.

We test the three algorithms on three conductivity models as illustrated in figure 5. All three models have the background conductivity equal to 1 . The first model contains a single inclusion with conductivity value equal to 2 ; the second contains two inclusions with conductivity values equal to 2 and $1 / 2$, respectively; and the third contains two inclusions, both with conductivity values equal to 2 .

\subsection{Noiseless Data}

We first consider noiseless data. Figure 6 displays an overhead view of the reconstructed images obtained for test conductivity 1 . In this test, the variational methods are able to produce images with superior resolution. The error plots associated with these reconstructions are shown in figure 7. They illustrate the progress of the relative error in $\sigma$, calculated in the Euclidean norm as

$$
\frac{\left\|\sigma^{*}-\sigma^{k}\right\|_{2}}{\left\|\sigma^{*}\right\|_{2}}
$$



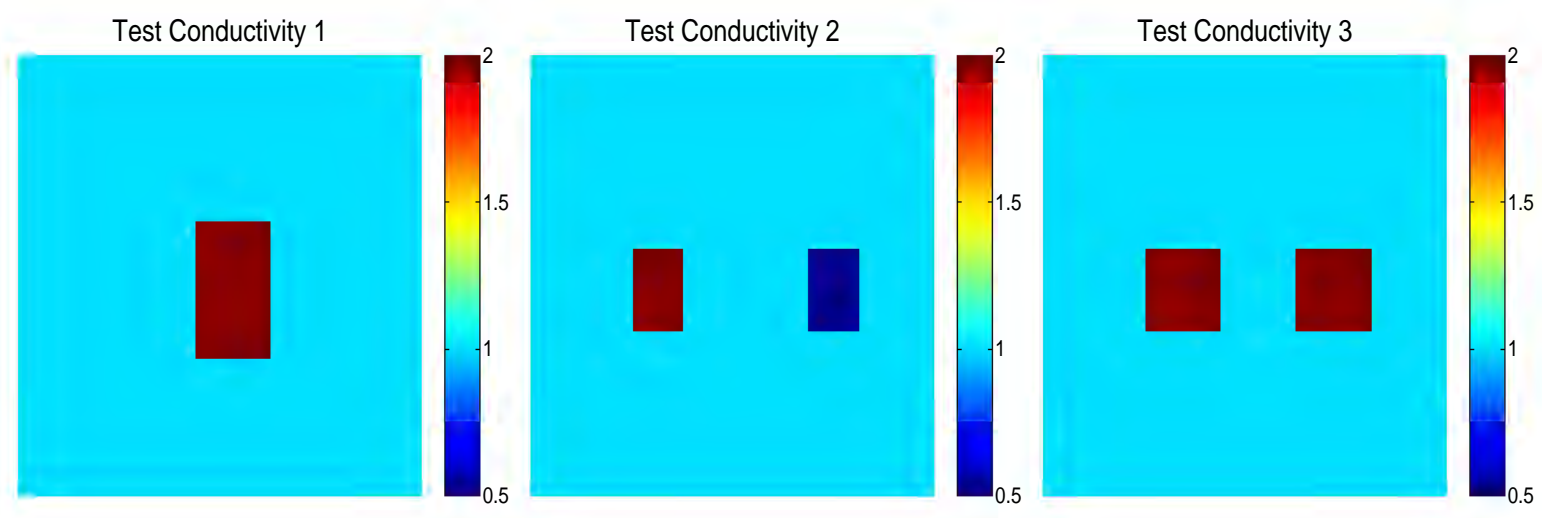

Figure 5: The three conductivities models used in the numerical study.

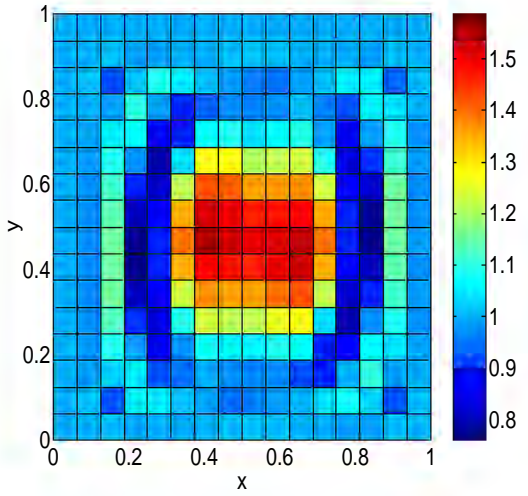

(a) Output least squares

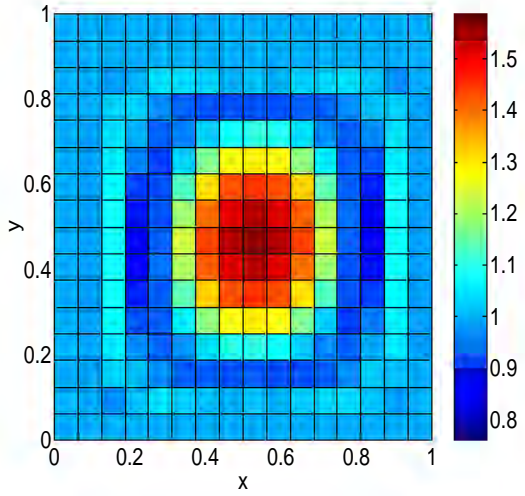

(b) Equation-error

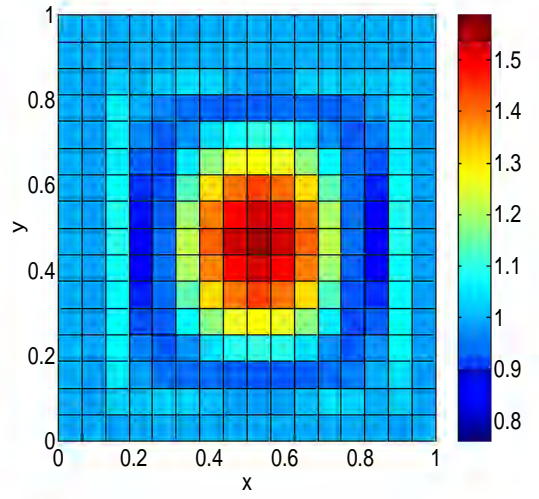

(c) Constrained least squares

Figure 6: Reconstructions of test conductivity 1 for noiseless data.

where $\sigma^{*}$ is the true conductivity and $\sigma^{k}$ is the computed conductivity at the $k$-th iteration, and the progress of the relative fit of the boundary data

$$
\sum_{e=1}^{N} \frac{\left\|\left(\Lambda_{\sigma^{k}}\right)^{-1} I_{e}-V_{e}\right\|_{L^{2}(\partial \Omega)}^{2}}{\left\|V_{e}\right\|_{L^{2}(\partial \Omega)}^{2}}
$$

In the case of the output least squares algorithm, the relative error eventually begins to increase despite the fact that the relative fit of the boundary data continues to decrease. In contrast, the variational algorithms continually reduce both the relative error and the relative fit of the boundary data. This fact seems to indicate that while devising a good stopping criterion for the output least squares algorithm is challenging, it may be less so for the variational algorithms. Finally, we note that for this test, the constrained least squares and equation-error methods perform equally well. We also note that the equation-error approach stops earlier than the other methods because it can no longer achieve the required relative change in its objective function. In contrast, the objective functions of the output least squares and constrained least squares algorithms continue to change and thus, these algorithms do not stop until they reach a maximum number of iterations. 

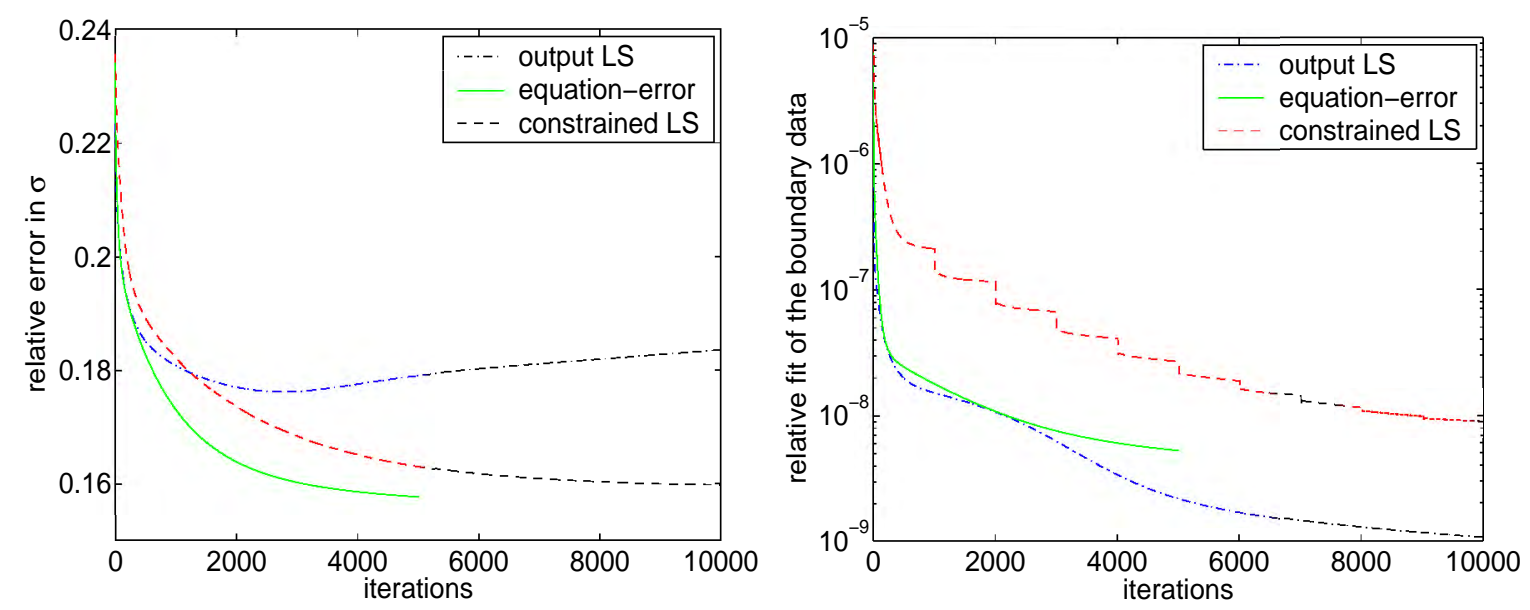

Figure 7: The graph on the left plots the relative error in $\sigma$, calculated as (6.1), and the graph on the right plots the relative fit of the boundary data, calculated as (6.2), for the reconstructions of test conductivity 1 shown in figure 6 . Note that as the output least squares algorithm progresses, the relative error of $\sigma$, in blue on the left, increases despite the fact that the fit of the boundary data, in blue on the right, is still decreasing.

\subsection{Regularization}

The variational constraints restrict the space of admissible conductivity functions and, as such, they may introduce some natural regularization to the problem. However, the ill posedness is not eliminated by the variational constraints, as one can see easily from well known counter examples (see for example [3]). Although the optimization algorithms that we have used provide some iterative regularization (through the choice of the step lengths), it is clear from the initial results that further regularization is needed for better performance ${ }^{6}$. We take a Tikhonov type regularization [91], where we add to each objective function the regularization term

$$
\alpha\|\nabla \sigma\|_{L^{2}(\Omega)}^{2},
$$

for a small, positive parameter $\alpha$. This ensures that $\sigma-\sigma^{0} \in H_{0}^{1}(\Omega)$, where $\sigma^{0}$ is the initial guess. Then, by the compact embedding $H_{0}^{1}(\Omega) \subset \subset L^{2}(\Omega)[1]$, we have that any sequence $\left\{\sigma^{k}\right\}$ of iterates, contains at least a subsequence $\left\{\sigma^{k_{l}}\right\}$ which converges strongly in $L^{2}(\Omega)$, to $\sigma_{\alpha}$, a minimizer of the regularized objective function.

Clearly, there are many other possible regularization methods (see for example $[9,39,40,49$, $51,70,73,91]$ and the references therein). Our investigation focuses on the performance of the variational formulations, rather than on the choice of regularization. Thus, we take a convenient regularization method, which is inexpensive and easy to implement.

The parameter $\alpha$ is chosen with the Morozov discrepancy principle [40,73,78], implemented numerically via the practical procedure suggested in [9]. We begin with a relatively large value for $\alpha$ and, as the algorithm progresses, we gradually decrease $\alpha$ until the value of the objective

\footnotetext{
${ }^{6}$ For example, if we examine the objective function near the computed solution, we observe the following manifestation of the ill-posedness of the EIT problem: Without additional regularization, the objective function appears to be very flat so that two conductivity distributions may attain approximately the same objective function value, but differ significantly from one another. The addition of a regularization term makes the function appear more "curved" and it thus reduces the severity of ill-conditioning.
} 
function is less than some specified tolerance $\varepsilon$. In the case of noiseless data, $\varepsilon$ reflects numerical error. When noise is present, the tolerance is chosen so as to avoid fitting the noise in the data. For both the output least squares and constrained least squares methods, the inequality to be satisfied is

$$
\sum_{e=1}^{N} \frac{\left\|\left(\Lambda_{\sigma}\right)^{-1} I_{e}-V_{e}\right\|_{L^{2}(\partial \Omega)}^{2}}{\left\|V_{e}\right\|_{L^{2}(\partial \Omega)}^{2}} \leq \varepsilon .
$$

Since the stopping criterion is the same for these two methods, we choose the same regularization parameter for both methods. For the equation-error method, $\alpha$ is reduced until

$$
\sum_{e=1}^{N} \frac{\left(\left\langle V_{e}, \Lambda_{\sigma} V_{e}\right\rangle+\left\langle I_{e},\left(\Lambda_{\sigma}\right)^{-1} I_{e}\right\rangle-2 P_{e}\right)}{2 P_{e}} \leq \varepsilon .
$$

Because the equation-error method has a very different objective functional, its regularization parameter $\alpha$ may differ from that of the output least squares and constrained least squares methods. Furthermore, it is unclear how to choose the tolerance $\varepsilon$ for the equation-error method. For the purposes of our comparison study, we compute the $\varepsilon$ in (6.5) by calculating the left hand side of (6.5) with both noiseless and noisy data for a known conductivity. Although this way of choosing the tolerance works only in simulation studies, we use it to ensure that we do our best to choose the parameter $\varepsilon$ for the equation-error method, in order to have a fair comparison.

We first consider the regularized formulations with noiseless data. The images of test conductivity 1 produced by each of the three algorithms are shown in figure 8 . Note that as expected, the output least squares algorithm is vastly improved. However, the inclusion is still relatively spread out. Both the constrained least squares and equation-error methods show some improvement as well. Their images are smoother and of better resolution and magnitude.

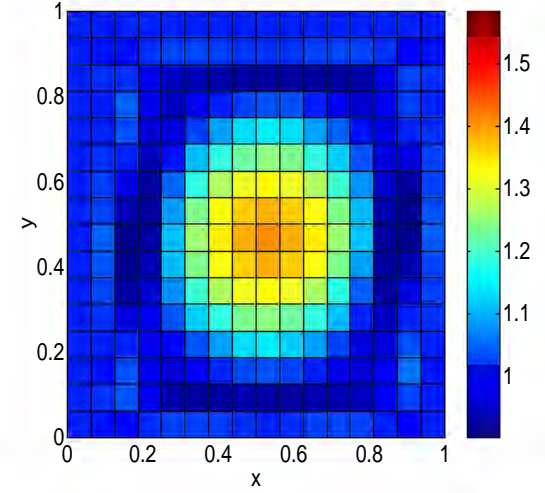

(a) Output least squares

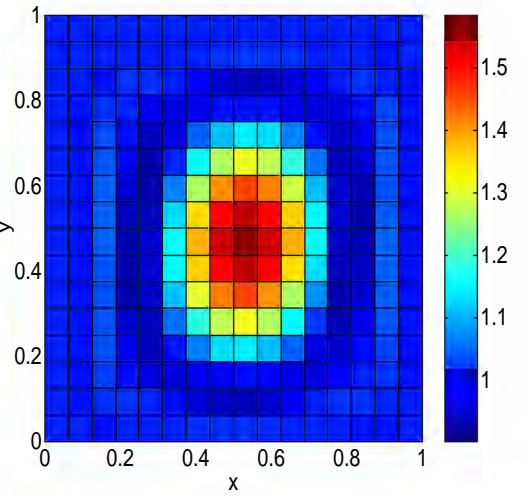

(b) Equation-error

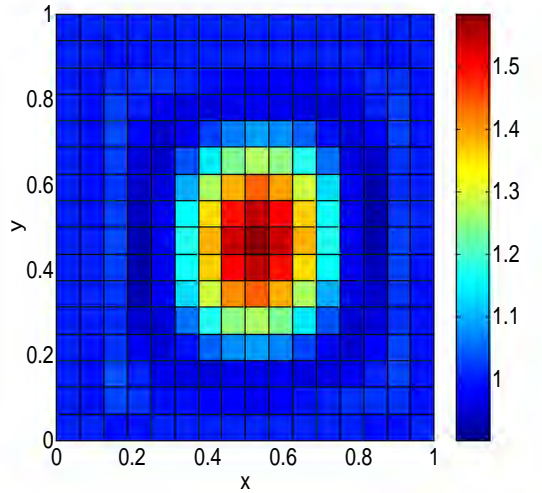

(c) Constrained least squares

Figure 8: Reconstructions of test conductivity 1 with the regularized methods for noiseless data.

We reiterate that the output least squares and the constrained least squares algorithms use the same regularization parameter $\alpha$. For this test, we begin with $\alpha=10^{-4}$ and reduce by a factor of 2 until it is approximately $10^{-6}$. The equation-error method begins with $\alpha=10^{-6}$ which is progressively reduced by two orders of magnitude. The relative error and relative fit of the boundary data plots are given in figure 9. With regularization, the relative error in $\sigma$ for the output least squares algorithm no longer increases. However, it is still significantly higher than the relative errors achieved by the variational methods despite the fact that all three methods fit the data on the boundary equally well. 

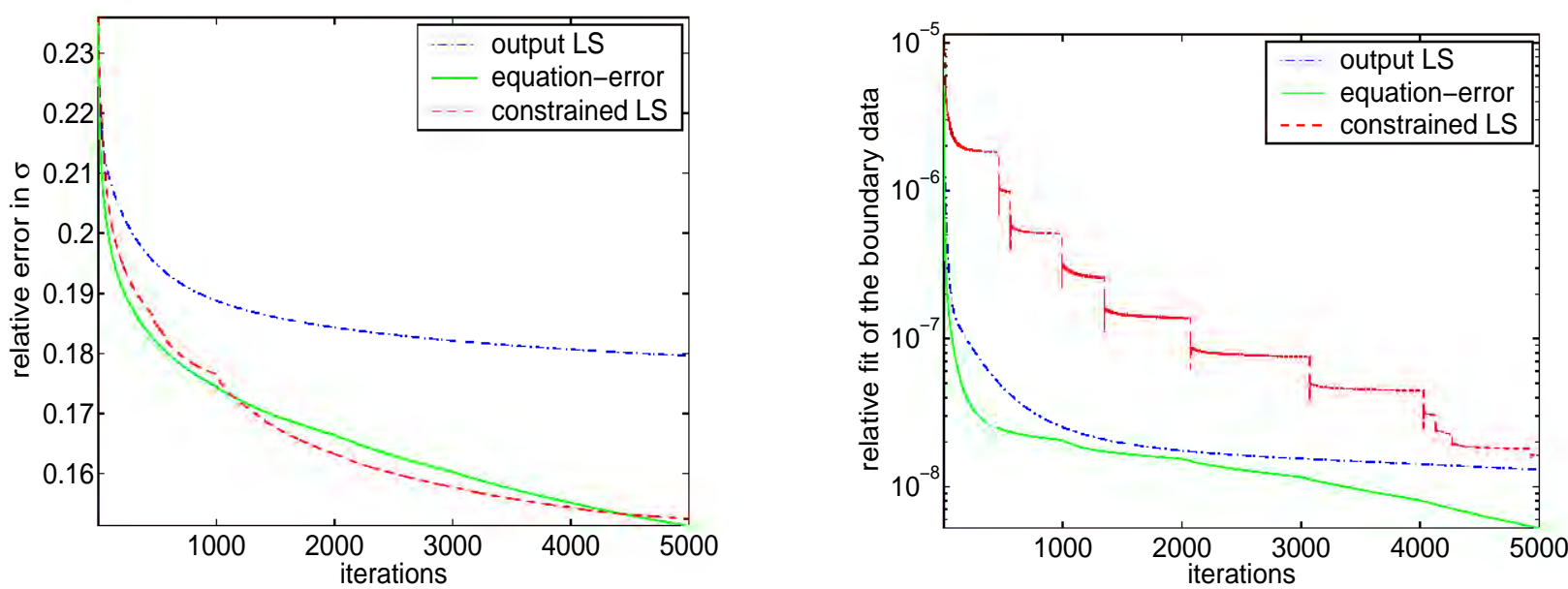

Figure 9: The plot on the left is the relative error in $\sigma$, as described in equation (6.1), achieved by the three regularized methods in reconstructing test conductivity 1 with noiseless data. The plot on the right shows the relative fit of the boundary data, calculated using equation (6.2) for the same test. Note that the relative error in the least squares algorithm is significantly higher than those of the variational methods despite the fact that all three methods fit the boundary data equally well.

The reconstructions of test conductivity 2 obtained with the regularized methods are displayed in figure 10. Here, both the output and constrained least squares methods begin with a regularization parameter of $\alpha=10^{-5}$. This is subsequently reduced approximately three orders of magnitude. The equation-error method reduces $\alpha$ to $10^{-9}$. Figure 11 depicts a three dimensional view of the

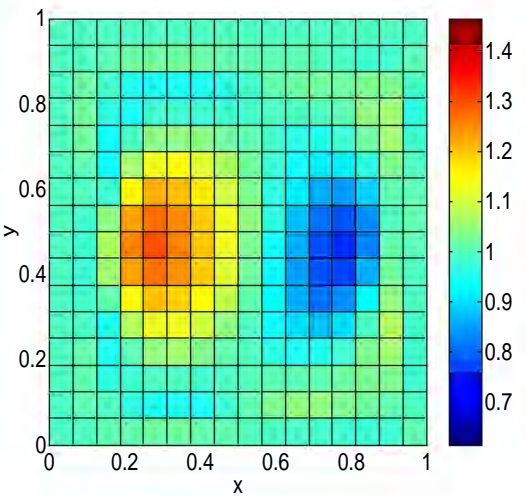

(a) Output least squares

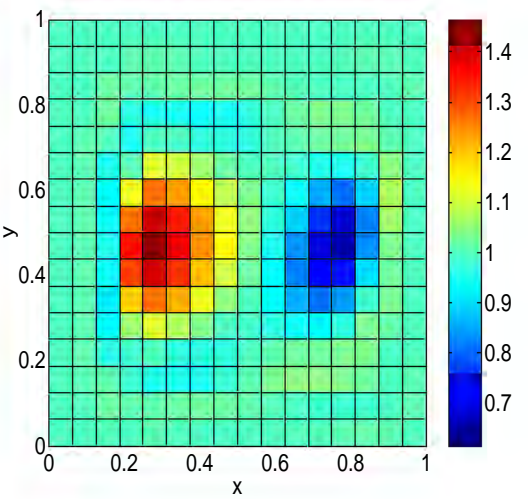

(b) Equation-error

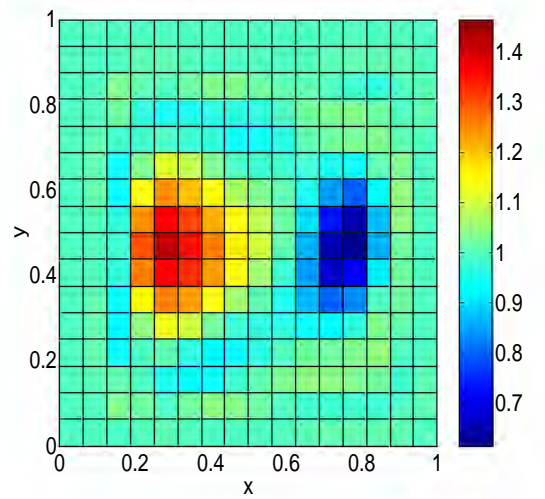

(c) Constrained least squares

Figure 10: Reconstructions of test conductivity 2 with the regularized methods for noiseless data.

reconstructed images of test conductivity 3 achieved with the regularized formulations. Again, the constrained least squares and equation-error methods produce comparable images, but the relative error in $\sigma$ is much higher for the regularized output least squares algorithm. For this test, all three methods are able to fit the data so that

$$
\sum_{e=1}^{N} \frac{\left\|\left(\Lambda_{\sigma}\right)^{-1} I_{e}-V_{e}\right\|_{L^{2}(\partial \Omega)}^{2}}{\left\|V_{e}\right\|_{L^{2}(\partial \Omega)}^{2}} \sim \mathcal{O}\left(10^{-8}\right) .
$$




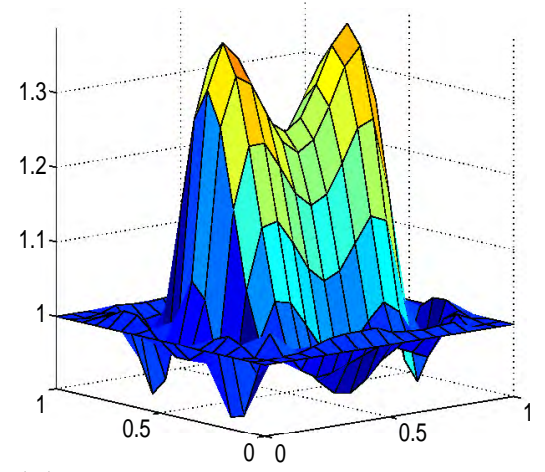

(a) Output least squares

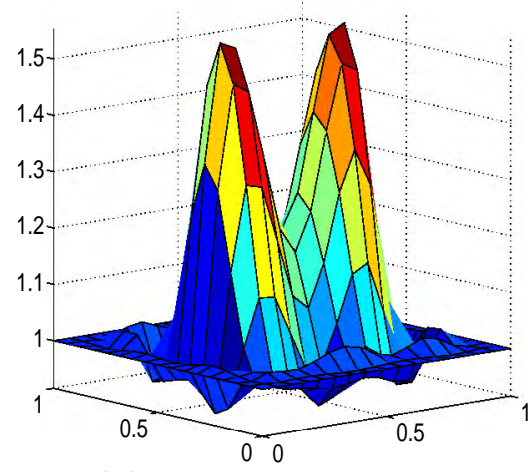

(b) Equation-error

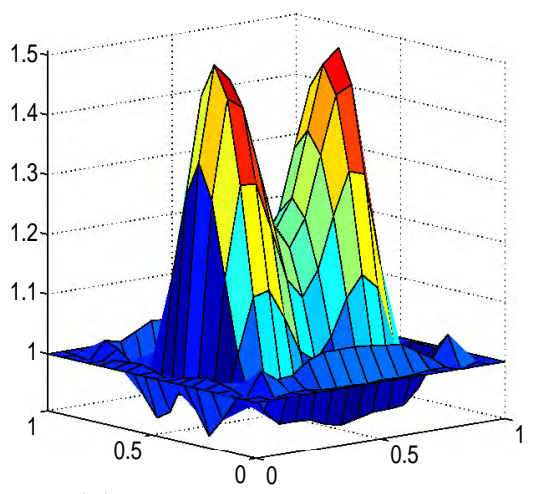

(c) Constrained least squares

Figure 11: Reconstructions of test conductivity 3 with the regularized methods for noiseless data.

Note that all images can be improved by starting with a better initial guess $\sigma^{0}$, than the constant $\sigma^{0}=1$ that we have considered here. See for example the results in $[17,48]$, where $\sigma^{0}$ is estimated by a multigrid approach.

\subsection{Noisy Data}

In practice, EIT data is contaminated with noise. Hence we test the three algorithms using noisy data at a $1 \%$ noise level and a 3\% noise level. Past studies have considered data with noise levels as high as $20 \%$ (see for example $[53,94]$ ). However, most algorithms tend to perform very poorly with such inaccurate data. Furthermore, with today's technology, it is expected that the noise level in EIT data be small ${ }^{7}$.

We add uniformly distributed, multiplicative random noise to each of the vectors of boundary potentials, $\mathbf{V}_{\mathbf{e}}$, for $e=1, \ldots, N$. Thus, for each experiment $e$, the voltage data is now

$$
V_{e}(i)=V_{e}(i)+\frac{\xi}{100} \varphi_{i}\left|V_{e}(i)\right|
$$

where $V_{e}(i)$ is the $i$-th component of vector $\mathbf{V}_{\mathbf{e}}$, corresponding to the $i^{\text {th }}$ boundary point, $\xi$ is the strength of the noise, and $\varphi_{i}$ is a random number generated by function RANDOM_NUMBER intrinsic to Fortran 90.

\subsubsection{Numerical Results with $1 \%$ Noise}

Figure 12 shows the reconstructions of test conductivity 1 obtained with data at a $1 \%$ noise level. As expected, these results are not as good as the noiseless ones. However, all three methods are still able to distinguish the inclusion. The image produced by the output least squares algorithm contains some spurious artifacts. Despite this, the resolution and magnitude of the inclusion are still quite good. The equation-error method also introduces some artifacts, but they are not as significant or as numerous. However, the magnitude of the inclusion is lower than that of the other two methods. Therefore, we can conclude that the image recovered by the constrained least

\footnotetext{
${ }^{7} \mathrm{~A}$ discussion of measurement accuracy in real data gathering experiments can be found, for example, in [79]. Given the present modern equipment, the noise level expected is around $1 \%$. Nevertheless, the number $1 \%$ is not always accurate and it may be site and application dependent. In particular, measurements for medical imaging applications [54] are usually more accurate than those in geophysics.
} 


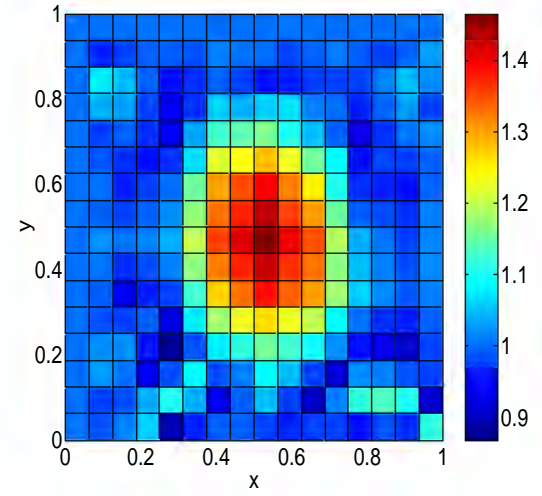

(a) Output least squares

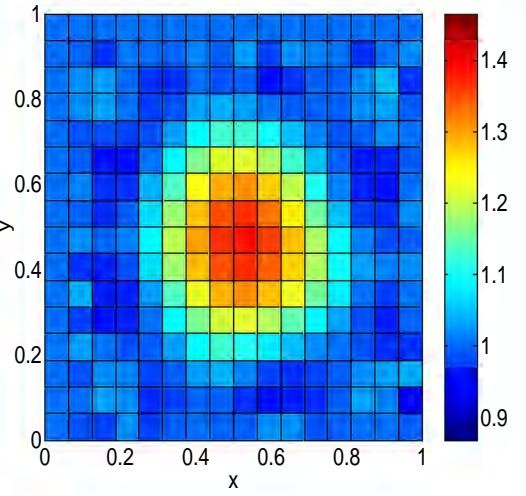

(b) Equation-error

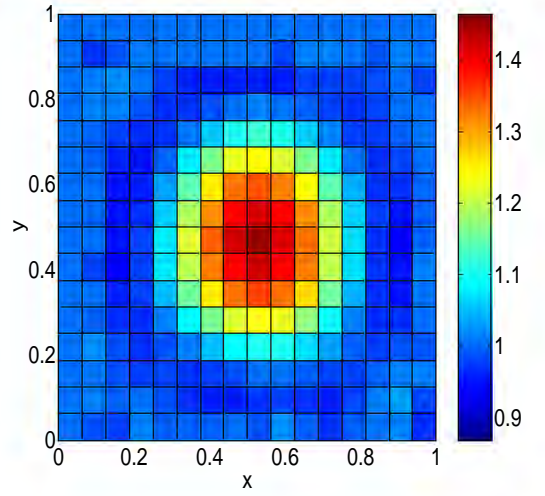

(c) Constrained least squares

Figure 12: Reconstructions of test conductivity 1. Noise level: 1\%.

squares method is superior in this case. It has good resolution and magnitude, it is smooth, and it contains fewer artifacts. Although the constrained and output least squares methods use the same regularization parameters, the constrained least squares algorithm produces a much smoother image.

Intuitively, we can better understand why the constrained least squares methods is superior in the presence of noise by examining the iterative process of the three methods. The output least squares and equation-error methods have no explicit restrictions on $\sigma$. Thus, the iterates are allowed to move freely. We observe that they exhibit a sort of "zig-zag" behavior pattern and do not stay in the same feasibility region from one iteration to the next. Thus, the iterates jump in and out of the Dirichlet and Thomson feasibility regions. This behavior can make the methods unstable, particularly in the presence of noise. In contrast, the constrained least squares method restricts the iterates to the Dirichlet infeasibility region. Hence, the constraint controls the change in $\sigma$ and does not allow the "zig-zag" behavior to occur and the feasibility constraint prevents the kind of instability present in other two methods.

The recovered images of test conductivity 2 are displayed in figure 13. All three methods achieve comparable resolution. However, the magnitudes obtained by the variational methods are better than those of the output least squares algorithm. Both the output least squares and equation-error methods show a few spurious artifacts while the constrained least squares method is smoother and contains only one major artifact. Again, we see that the two least squares methods use the same regularization parameter with different results.

The reconstructed images of test conductivity 3 are given in figure 14 .

\subsubsection{Numerical Results with $3 \%$ Noise}

We repeat the reconstructions using regularization and the coarse grid initial guess with data containing 3\% noise. Figure 15 shows the reconstructed images of test conductivity 1 . Here, the output least squares is quite unsuccessful. The inclusion is very blurry and numerous artifacts are introduced, particularly near the boundary. This sort of behavior usually indicates the need for more regularization. However, with the same regularization, the constrained least squares method produces a smooth reconstruction without significant artifacts. The inclusion is still of reasonable resolution and magnitude. The constrained least squares method also outperforms the equationerror method in terms of resolution.

The images recovered for test conductivity 2 are displayed in figure 16 . In this case, the 


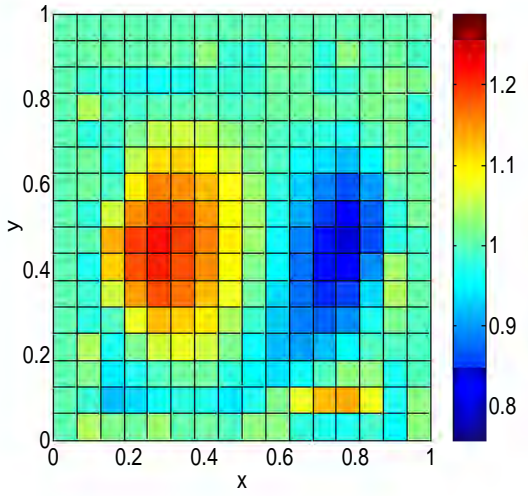

(a) Output least squares

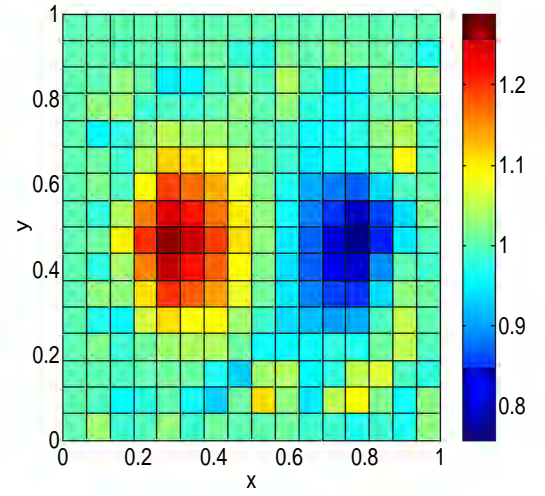

(b) Equation-error

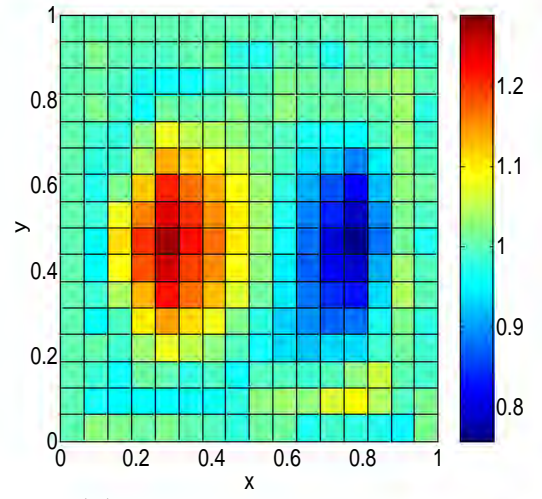

(c) Constrained least squares

Figure 13: Reconstructions of test conductivity 2. Noise level: 1\%.

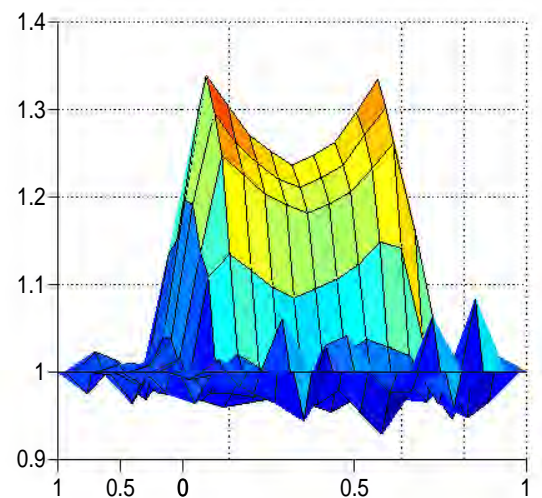

(a) Output least squares

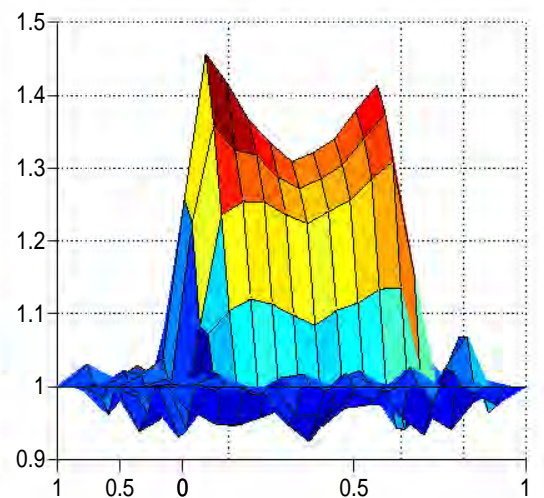

(b) Equation-error

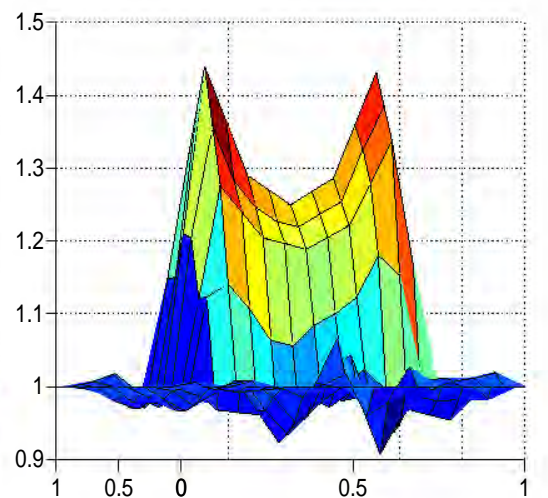

(c) Constrained least squares

Figure 14: Reconstructions of test conductivity 3. Noise level: 1\%.

output least squares method produces a relatively smooth image, but the magnitudes are very low. The equation-error method achieves much better magnitudes but introduces many artifacts near the boundary. The constrained least squares combines the best features of the other two reconstructions. It produces an image that is quite smooth and of reasonable magnitude without introducing many artifacts.

\subsection{Summary of the Numerical Results}

Based on the presented numerical results, we make the following observations on the performance of the three methods on the set of test cases.

- The constrained least squares and equation-error variational methods are superior to output least squares. Without noise in the data, the equation-error and constrained least squares methods perform equally well. In the presence of noise, the constrained least squares method appears to be better.

- The output least squares method fits the boundary data in the $L^{2}$ norm as well as, or better than the constrained least squares and equation-error methods. However, the variational methods produce better images. 


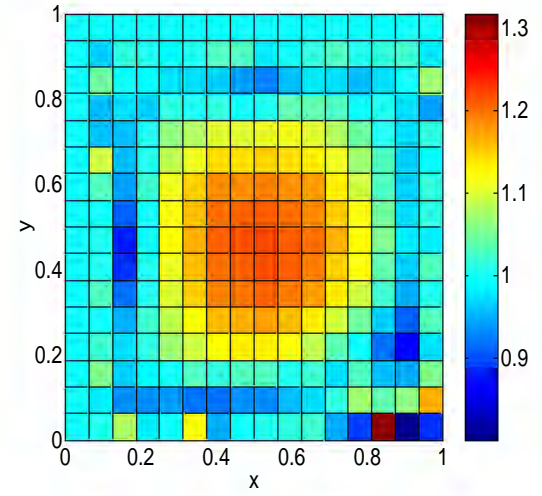

(a) Output least squares

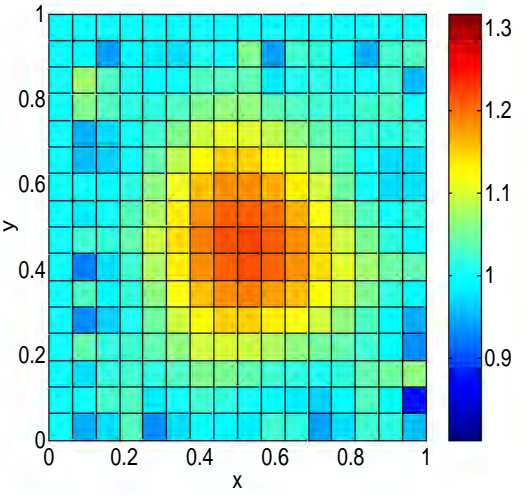

(b) Equation-error

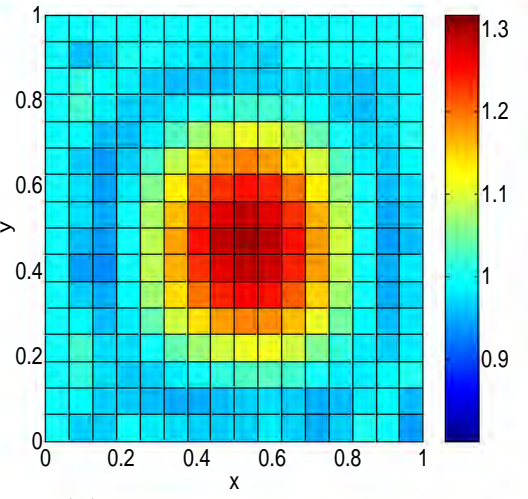

(c) Constrained least squares

Figure 15: Reconstructions of test conductivity 3. Noise level: $3 \%$

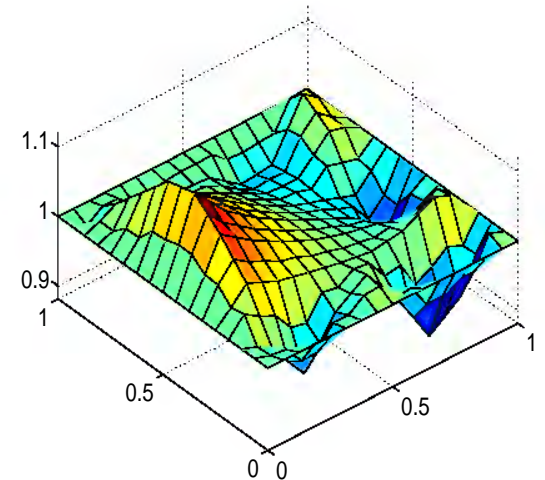

(a) Output least squares

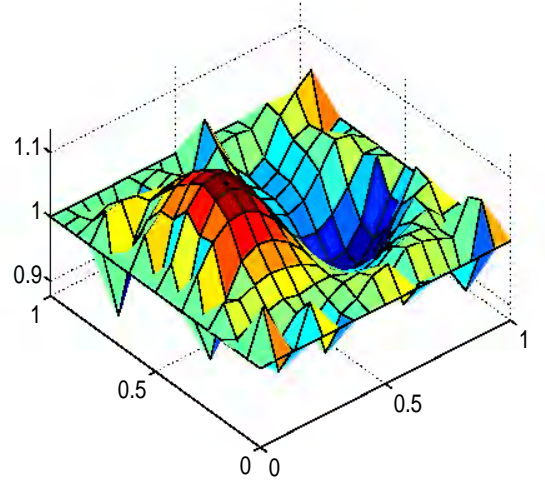

(b) Equation-error

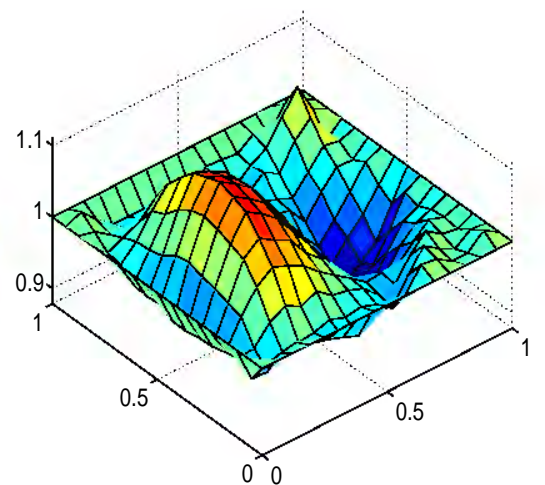

(c) Constrained least squares

Figure 16: Reconstructions of test conductivity 2. Noise level: $3 \%$

- In the presence of noise and with the same regularization parameter, the constrained least squares method is able to produce smoother images with less spurious artifacts than the output least squares is.

\section{Summary}

We have introduced a set of variationally constrained reconstruction methods for electrical impedance tomography. The Dirichlet and Thomson variational constraints on the conductivity function $\sigma$ have been previously introduced by Berryman and Kohn in [14]. In this paper, we analyze the constraints and show how to used them efficiently in inversion. We discuss a variety of algorithms and describe in detail the implementation and the performance of one of them - the constrained least squares approach. Comparisons with two well-known methods, the unconstrained least squares (see for example [94]) and the equation-error [66,93], are given. Traditionally, the unconstrained least squares approach has been the method of choice, due to its simplicity and relatively low computational cost. However, the unconstrained least squares approach does not make the best use of the measured data (i.e., boundary voltage $V=\left(\Lambda_{\sigma^{\star}}\right)^{-1} I$ ) because it fits the data in the convenient $L^{2}(\partial \Omega)$-norm, instead of the more natural and stronger $H^{1 / 2}(\partial \Omega)$-norm. We have shown that variational constraints can be used efficiently to achieve a better fit and that at the reconstructed $\sigma$ 
the current density $\mathbf{j}$, satisfying the Neumann boundary conditions $-\left.\mathbf{j} \cdot \mathbf{n}\right|_{\partial \Omega}=I$, and the potential $\phi$, satisfying the Dirichlet boundary conditions $\left.\phi\right|_{\partial \Omega}=V$, are correctly related by Ohm's law $\mathbf{j}=-\sigma \nabla \phi$. We show that achieving such a data fit can be done with acceptable computational cost and that the resulting images of $\sigma$ can have better resolution than those generated by conventional, unconstrained least squares methods.

\section{Acknowledgments}

The work of L. Borcea was partially supported by the National Science Foundation under grant number DMS-9971209 and by the Office of Naval Research, under grant N00014-02-1-0088. The work of G. A. Gray was supported by a training fellowship of the Keck Center for Computational Biology (National Library of Medicine Training Grant No. 1T15LM07093). The work of Y. Zhang was partially supported by DOE Grant DE-FG03-97ER25331, DOE/LANL Contract 03891-99-23 and NSF Grant DMS-9973339.

\section{References}

[1] R. A. Adams. Sobolev Spaces. Academic Press, Inc., New York, 1975.

[2] M. R. Akbarzadeh, W. J. Tompkins, and J. G. Webster. Multichannel impedance pneumography for apnea monitoring. Proc. Annu. Int. Conf. IEEE Eng. Med. Biol. Soc., 12:1048-1049, 1990.

[3] G. Alessandrini. Stable determination of conductivity by boundary measurements. Applicable Analysis, 27:153-172, 1988.

[4] G. Alessandrini. Stable determination of conductivity by boundary measurements. App. Anal., 27:153-172, 1988.

[5] G. Alessandrini, E. Beretta, F. Santosa, and S. Vessella. Stability in crack determination from electrostatic measurements at the boundary-a numerical investigation. Inverse Problems, 11(5):L17-L24, 1995.

[6] G. Alessandrini and L. Rondi. Stable determination of a crack in a planar inhomogeneous conductor. SIAM J. Math. Anal., 30(2):326-340, 1998.

[7] A. Allers and F. Santosa. Stability and resolution analysis of a linearized problem in electrical impedance tomography. Inverse Problems, 7:515-513, 1991.

[8] M. Arioli, J.W. Demmel, and I.S. Duff. Solving sparse linear systems with sparse backward error. SIAM J. Matrix Anal. Appl., 10(2):165-190, April 1989.

[9] U. M. Ascher and E. Haber. Grid refinement and scaling for distributed parameter estimation problems. Inverse Problems, 17:571-590, 2001.

[10] D. Barber and B. Brown. Applied potential tomography. J. Phys. E. Sci. Instrum., 17:723-733, 1984 .

[11] D.C. Barber and B.H. Brown. Progress in electrical impedance tomography. In Colton, Ewing, and Rundell, editors, Inverse Problems in Partial Differential Equations. SIAM, Philadelphia, PA, 1990. 
[12] C. A. Berenstein and E. Casadio Tarabusi. Integral geometry in hyperbolic spaces and electrical impedance tomography. SIAM J. Appl. Math., 56(3):755-764, 1996.

[13] J. G. Berryman. Convexity properties of inverse problems with variational constraints. Journal of the Franklin Institute, 328(1):1-13, 1991.

[14] J. G. Berryman and R. V. Kohn. Variational constraints for electrical impedance tomography. Phys. Rev. Lett., 65:325-328, 1990.

[15] J.G. Berryman. Lecture notes on nonlinear inversion and tomography: II. Electrical impedance tomography. Presented at U.C. Berkeley in 1992.

[16] J.G. Berryman. Stable iterative reconstruction algorithm for nonlinear traveltime tomography. Inverse Problems, 6:21-42, 1990.

[17] L. Borcea. Nonlinear multigrid for imaging electrical conductivity and permittivity at low frequency. Inverse Problems, 17(2):329-359, 2001.

[18] L. Borcea. Electrical impedance tomography. Inverse Problems, 18(4):R99-R136, 2002.

[19] L. Borcea, J. G. Berryman, and G. C. Papanicolaou. Matching pursuit for imaging high contrast conductive media. Inverse Problems, 15(4):811-849, 1999.

[20] L. Borcea, J.G. Berryman, and G.C. Papanicolaou. Matching pursuit for imaging high contrast conductive media. Inverse Problems, 15(12):811-849, 1999.

[21] R. M. Brown and G. Uhlmann. Uniqueness in the inverse conductivity problem for nonsmooth conductivities in two dimensions. Comm. Partial Differential Equations, 22(5-6):1009-1027, 1997.

[22] M. Brühl and M. Hanke. Numerical implementation of two noniterative methods for locating inclusions by impedance tomography. Inverse Problems, 16:1029-1042, 2000.

[23] A.P. Calderón. On an inverse boundary value problem. In Seminar on Numerical Analysis and its Applications to Continuum Physics, pages 65-73, Rio de Janeiro, 1980. Soc. Brasileira de Matèmatica.

[24] D. J. Cedio-Fengya, S. Moskow, and M. S. Vogelius. Identification of conductivity imperfections of small diameter by boundary measurements. continuous dependence and computational reconstruction. Inverse Problems, 14:553-595, 1998.

[25] G. Chavent and K. Kunisch. Regularization in state space. J. Numer. Anal., 27:535-564, 1993.

[26] M. Cheney and D. Isaacson. Distinguishability in impedance imaging. IEEE Trans. Biomed. Eng., 39(8):852- 860, 1992.

[27] M. Cheney, D. Isaacson, and J.C. Newell. Electrical impedance tomography. SIAM Review, 41(1):85-101, 1999.

[28] R. Courant and D. Hilbert. Methods of Mathematical Physics, volume 1. Wiley, New York, 1953.

[29] J.W. Demmel, S.C. Eisenstat, J.R. Gilbert, X.S. Li, and J.W.H. Liu. A supernodal approach to sparse partial pivoting. SIAM J. Matrix Anal. Appl., 20(3):720-755, 1999. 
[30] J. E. Dennis and R. B. Schnabel. Numerical Methods for Unconstrained Optimization and Nonlinear Equations. SIAM, Philadelphia, 1996.

[31] D. C. Dobson. Estimates on resolution and stabilization for the linearized inverse conductivity problem. Inverse Problems, 8(1):71-81, 1992.

[32] D.C. Dobson. Stability and Regularity of an Inverse Elliptic Boundary Value Problem. PhD thesis, Rice University, April 1990.

[33] D.C. Dobson. Convergence of a reconstruction method for the inverse conductivity problem. SIAM J. Appl. Math., 1992.

[34] D.C. Dobson and F. Santosa. Resolution and stability analysis of an inverse problem in electrical impedance tomography: dependence on the input current patterns. SIAM J. Appl. Math., 54(6):1542-1560, 1994.

[35] V. Druskin. The unique solution of the inverse problem of electrical surveying and electrical well-logging for piecewise-continuous conductivity. Earth Physics, 18:51-53, 1982.

[36] V. Druskin. On uniqueness of the determination of the three-dimensional underground structures from surface measurements with variously positioned steady-state or monochromatic field sources. Soviet Physics of the Solid Earth (English translation by American Geophysical Union), 21(3):210-214, 1985.

[37] M. R. Eggleston, R. J. Schwabe, D. Isaacson, and L. F. Coffin. The application of electric current computed tomography to defect imaging in metals. Review of Progress in Quantitative NDE, D. O. Thompson and D. E. Chimenti Eds. Plenum, New York, 1989.

[38] I. Ekeland and R. Témam. Convex analysis and variational problems. SIAM, Philadelphia, 1999.

[39] H. Engl. Regularization methods for the stable solution of inverse problems. Surv. Math. Ind., 3:71-143, 1993.

[40] H. W. Engl, M. Hanke, and A. Neubauer. Regularization of Inverse Problems. Kluwer Academic Publishers, Dodrecht, 1996.

[41] H. W. Engl, K. Kunisch, and A. Neubauer. Convergence rates for tikhonov regularization of nonlinear ill-posed problems. Inverse Problems, 5:523-540, 1989.

[42] A.V. Fiacco and G.P. McCormick. Nonlinear Programming: Sequential Unconstrained Minimization Techniques. SIAM, Philadelphia, PA, 1990.

[43] G. B. Folland. Introduction to partial differential equations. Princeton University Press, Princeton, N.J., 1995.

[44] P. Colli Franzone, L. Guerri, B. Taccardi, and C. Viganotti. The direct and inverse potential problems in electrocardiology. Numerical aspects of some regularization methods and application to data collected in isolated dog heart experiments. Pub. 222, Laboratorio di Analisi Numerica del Consiglio Nazionale delle Richerche, Pavia, 1979.

[45] A. Friedman and M. Vogelius. Determining cracks by boundary measurements. Indiana Univ. Math. J., 3:527-556, 1989. 
[46] D. G. Gisser, D. Isaacson, and J. C. Newell. Electric current computed tomography and eigenvalues. SIAM J. Appl. Math., 50(6):1623-1624, 1990.

[47] G.H. Golub and C.F. Van Loan. Matrix Computations. The Johns Hopkins University Press, Baltimore, MD, third edition, 1996.

[48] G. Gray. A variationally constrained numerical solution of the electrical impedance tomography problem,. In Computational and Applied Mathematics, Rice University, PhD thesis, Technical Report: 02-02, 2002.

[49] E. Haber and U. Ascher. Preconditioned all-at-once methods for large, sparse parameter estimation problems. Inverse Problems, 17:1847-1864, 2001.

[50] E. Haber, U. Ascher, and D. Oldenburg. On optimization techniques for solving nonlinear inverse problems. Inverse Problems, 16:1263-1280, 2000.

[51] M. Hanke. Regularizing properties of a truncated newton-cg algorithm for nonlinear inverse problems. Numer. Funct. Anal. Optimiz., 18:971-993, 1997.

[52] N.D. Harris, A.J. Suggett, D.C. Barber, and B.H. Brown. Applications of applied potential tomography (ap) in respiratory medicine. Clin. Phys. Physiol. Meas., 8(A):155-165, 1987.

[53] B. Hofmann. Approximation of the inverse electrical impedance tomography problem by an inverse transmission problem. Inverse Problems, 14:1171-1187, 1998.

[54] D. Holder. Clinical and physiological applications of electrical impedance tomography. UCL Press, London, 1993.

[55] D. Isaacson. Distinguishabiltiy of conductivities by electric current computed tomography. IEEE Trans. Biomed. Eng., MI-5(2):91-95, 1986.

[56] D. Isaacson and M. Cheney. Current problems in impedance imaging. Inverse Problems in Partial Differential Equations, D. Colton, R. Ewing and W. Rundell eds., SIAM, Philadelphia, pages 141-149, 1990.

[57] V. Isakov. Inverse problems for partial differential equations. Springer-Verlag, New York, 1998.

[58] J.D. Jackson. Classical Electrodynamics. Wiley, New York, second edition, 1974.

[59] V. V. Jikov, S. M. Kozlov, and O. A. Oleinik. Homogenization of differential operators and integral functionals. Springer-Verlag, Berlin, Heidelberg, New York, 1991.

[60] C. Johnson. Inverse bioelectric field problems: Modeling, simulation, and visualization. Invited lecture at the SFB-Conference on "Computational Methods for Inverse Problems" in Strobl, Austria, August, 25 - 31, 2002.

[61] J. P. Kaipio, V. Kolehmainen, E. Somersalo, and M. Vauhkonen. Statistical inversion and monte carlo sampling methods in electrical impedance tomography. Inverse Problems, 16:14871522, 2000.

[62] J. P. Kaipio, V. Kolehmainen, M. Vauhkonen, and E. Somersalo. Inverse problems with structural prior information. Inverse Problems, 15:713-729, 1999. 
[63] J.P. Kaipio, V. Kolehmainen, E. Somersalo, and M. Vauhkonen. Statistical inversion and Monte Carlo sampling in electrical impedance tomography. Inverse Problems, 16(5):1487-1522, 2000.

[64] B. Kaltenbacher. On the regularizing properties of a full multigrid method for ill-posed problems. Inverse Problems, 17:767-788, 2001.

[65] G. V. Keller. Electrical properties of rocks and minerals, Handbook of physical constants, S. P. Clarck Jr. ed., 553-577. Geological Society of America, New York, 1988.

[66] R. V. Kohn and A. McKenney. Numerical implementation of a variational method for electrical impedance tomography. Inverse Problems, 6:389-414, 1990.

[67] R. V. Kohn and M. Vogelius. Determining conductivity by boundary measurements. Comm. Pure App. Math., 37:113-123, 1984.

[68] R. V. Kohn and M. Vogelius. Determining conductivity by boundary measurements ii. interior results. Comm. Pure App. Math., 38:643-667, 1985.

[69] R. V. Kohn and M. Vogelius. Relaxation of a variational method for impedance computed tomography. Comm. Pure App. Math., XL:745-777, 1987.

[70] K. Kunisch and J. Zou. Iterative choices of regularization parameters in linear inverse problems. Inverse Problems, 14:1247-1264, 1998.

[71] S. Luo, V.X. Afonso, J.G. Webster, and W.J. Tompkins. The electrode system in impedancebased ventilation measurements. IEEE Trans. Biomed. Eng., 39(11):1130-1141, Nov. 1992.

[72] V. A. Morozov. Methods for solving Incorrectly posed problems. Springer-Verlag, New York, Berlin, Heidelberg, 1984.

[73] V.A. Morozov. On the solution of functional equations by the method of regularization. Doklady, 167(3):414-417, 1966.

[74] A. I. Nachman. Global uniqueness for a two-dimensional inverse boundary problem. Annals of Mathematics, 143:71-96, 1996.

[75] F. O'Sullivan. A statistical perspective on ill-posed inverse problems. Statistical Science, 1(4):502-527, 1986.

[76] Päivärinta, A. Panchencko, and G. Uhlmann. Complex geometrical optics solutions for lipschitz conductivities. Revista Matematica Iberoamericana, to appear, 2002.

[77] R. L. Parker. The inverse problem of resistivity sounding. Geophysics, 142:2143-2158, 1984.

[78] R.L. Parker. Geophysical Inverse Theory. Princeton University Press, Princeton, NJ, 1994.

[79] A. Ramirez, W. Daily, A. Binley, D. LaBrecque, and D. Roelant. Detection of leaks in underground storage tanks using electrical resistance methods. J. Env. and Eng. Geophysics, 1:189-203, 1996.

[80] A. Ramirez, W. Daily, D. LaBrecque, E. Owen, and D. Chestnut. Monitoring an underground steam injection process using electrical resistance tomography. Water Resources Research, 29:73-87, 1993. 
[81] F. Santosa, P. Kaup, and M. Vogelius. A method for imaging corrosion damage in thin plates from electrostatic data. Inverse Problems, 12:279-293, 1996.

[82] F. Santosa and M. Vogelius. A backprojection algorithm for electrical impedance imaging. SIAM J. Appl. Math., 50(1):216-243, 1990.

[83] F. Santosa and M. Vogelius. A computational algorithm for determining cracks from electrostatic boundary measurements. Int. J. Eng. Science, 29:917-938, 1991.

[84] H. P. Schwan and C. F. Kay. The conductivity of living tissues. Ann. NYAS, 65:1007-1013, 1957.

[85] A. Seagar. Probing with low frequency electric currents. Ph.D. Thesis, University of Canterbury, UK, 1983.

[86] S. Siltanen, J. Mueller, and D. Isaacson. An implementation of the reconstruction algorithm of a. nachman for the 2d inverse conductivity problem. Inverse Problems, 16(3):681-699, 2000.

[87] E. Somersalo, M. Cheney, and D. Isaacson. Existence and uniqueness for electrode models for electric current computed tomography. SIAM J. Appl. Math., 52(4):1023-1040, 1992.

[88] J. Sylvester and G. Uhlmann. A global uniqueness theorem for an inverse boundary value problem. Annals of Mathematics, 125:153-169, 1987.

[89] J. Sylvester and G. Uhlmann. The Dirichlet to Neumann map and applications. In Colton, Ewing, and Rundell, editors, Inverse Problems in Partial Differential Equations. SIAM, Philadelphia, PA, 1990.

[90] A. N. Tikhonov and V. Y. Arsenin. Solutions of Ill-Posed Problems, Translation editor Fritz John. John Wiley \& Sons, Washington DC, 1977.

[91] A.N. Tikhonov, A.S. Leonov, and A.G. Yagola. Nonlinear Ill-posed Problems, volume 1 and 2 of Applied Mathematics and Mathematical Computation. Chapman \& Hall, Eng. lang. edition, 1998.

[92] G. Uhlmann. Developments in inverse problems since Calderón's foundational paper. In Harmonic analysis and partial differential equations, Chicago Lectures in Math, pages 295345. Univ. Chicago Press, Chicago, IL, 1999.

[93] A. Wexler, B. Fry, and M. Neuman. Impedance-computed tomography algorithm and system. Appl. Opt., 24:3985-3982, 1985.

[94] T. J. Yorkey, J. G. Webster, and W. J. Tompkins. Comparing reconstruction algorithms for electrical impedance tomography. IEEE Trans. Biomed. Eng., BME-34(11):843-852, 1987. 